\title{
Deep-Sea Actinobacteria Mitigate Salinity Stress in Tomato Seedlings and Their Biosafety Testing
}

\author{
Pharada Rangseekaew ${ }^{1,2}$, Adoración Barros-Rodríguez ${ }^{3}$, Wasu Pathom-aree ${ }^{4, *}$ and Maximino Manzanera ${ }^{3} \mathbb{D}$ \\ 1 Doctor of Philosophy Program in Applied Microbiology (International Program) in Faculty of Science, \\ Chiang Mai University, Chiang Mai 50200, Thailand; Pharada_ra@cmu.ac.th \\ 2 Graduate School, Chiang Mai University, Chiang Mai 50200, Thailand \\ 3 Department of Microbiology, Institute for Water Research, University of Granada, 18071 Granada, Spain; \\ dorysbr@correo.ugr.es (A.B.-R.); manzanera@ugr.es (M.M.) \\ 4 Research Center of Microbial Diversity and Sustainable Utilization, Department of Biology, Faculty of Science, \\ Chiang Mai University, Chiang Mai 50200, Thailand \\ * Correspondence: wasu.p@cmu.ac.th; Tel.: +66-53943346-48
}

Citation: Rangseekaew, P.; Barros-Rodríguez, A.; Pathom-aree, W.; Manzanera, M. Deep-Sea Actinobacteria Mitigate Salinity Stress in Tomato Seedlings and Their Biosafety Testing. Plants 2021, 10, 1687. https://doi.org/10.3390/ plants10081687

Academic Editors:

Chrystalla Antoniou, Raffaella

Maria Balestrini and

Vasileios Fotopoulos

Received: 1 July 2021

Accepted: 11 August 2021

Published: 17 August 2021

Publisher's Note: MDPI stays neutral with regard to jurisdictional claims in published maps and institutional affiliations.

\begin{abstract}
Soil salinity is an enormous problem affecting global agricultural productivity. Deep-sea actinobacteria are interesting due to their salt tolerance mechanisms. In the present study, we aim to determine the ability of deep-sea Dermacoccus (D. barathri MT2.1 $1^{\mathrm{T}}$ and D. profundi $\mathrm{MT} 2.2^{\mathrm{T}}$ ) to promote tomato seedlings under $150 \mathrm{mM} \mathrm{NaCl}$ compared with the terrestrial strain $D$. nishinomiyaensis DSM20448 ${ }^{\mathrm{T}}$. All strains exhibit in vitro plant growth-promoting traits of indole-3-acetic acid production, phosphate solubilization, and siderophore production. Tomato seedlings inoculated with D. barathri MT2.1 $1^{\mathrm{T}}$ showed higher growth parameters (shoot and root length, dry weight, and chlorophyll content) than non-inoculated tomato and the terrestrial strain under $150 \mathrm{mM} \mathrm{NaCl}$. In addition, hydrogen peroxide $\left(\mathrm{H}_{2} \mathrm{O}_{2}\right)$ in leaves of tomatoes inoculated with deep-sea Dermacoccus was lower than the control seedlings. This observation suggested that deep-sea Dermacoccus mitigated salt stress by reducing oxidative stress caused by hydrogen peroxide. D. barathri MT2.1 $1^{\mathrm{T}}$ showed no harmful effects on Caenorhabditis elegans, Daphnia magna, Eisenia foetida, and Escherichia coli MC4100 in biosafety tests. This evidence suggests that D. barathri MT2.1 $1^{\mathrm{T}}$ would be safe for use in the environment. Our results highlight the potential of deep-sea Dermacoccus as a plant growth promoter for tomatoes under salinity stress.
\end{abstract}

Keywords: biosafety; Dermacoccus; marine actinobacteria; plant growth promotion; salt stress; sustainable agriculture

\section{Introduction}

Marine ecosystems cover more than $70 \%$ of the surface of the Earth, with most parts still under-explored [1]. The deep-sea is still poorly investigated in terms of microbial diversity due to the difficulty in obtaining the samples. Nevertheless, actinobacteria are widely distributed in deep-sea environments [2-4]. These marine actinobacteria are considered an excellent source of bioactive compounds. The adaptation of deep-sea microorganisms is interesting from an academic perspective and for their potential applications in biotechnology. The deep-sea environment is generally characterized by high pressure, low temperature, lack of light with varying concentrations of oxygen and salinity [2,5]. Deep-sea actinobacteria have to adapt or develop unique abilities to withstand salinity and pressure [6] or piezotolerant properties [7] to survive under these extreme conditions. With the adaptive ability to survive under extreme environments, especially salinity and osmotic stress, these deep-sea actinobacteria are likely to be useful as biostimulants to mitigate salinity stress for plant growth.

Salinity stress is considered a major problem causing a massive reduction in agricultural production worldwide. The total area of global cultivated land is $1.5 \times 10^{9}$ hectares; 
among these, $23 \%$ are saline soil and $37 \%$ sodic soil, between $25 \%$ and $30 \%$ of irrigated lands are also affected by salt [8]. Salinization impact on irrigated land is estimated to cost at least 12 billion US\$ in the annual global income $[9,10]$. High salinity damages plant growth and development mainly by ionic and osmotic stress [11,12]. Ionic stress leads to an excessive influx of sodium ions, resulting in an overall efflux of potassium ions [12-15]. Osmotic stress causes the accumulation of salts in the soil surrounding plant roots and leads to low water potential in soils. Hence, plants suffer from water and nutrients uptake interruption [6,16-19]. Salinity stress alters several plant morphological traits, such as a reduction in the number of leaves, plant size, roots length, and flower appearance [20-23]. Moreover, salinity induces the production of reactive oxygen species (ROS) causing a direct injury to plant tissues [16,24-26] and secondary DNA damages, such as base loss, DNA-protein crosslinks, and double-stranded DNA breakage [12,27]. In addition, the gradual accumulation of salts in plant parts damages cell membrane integrity and decreases membrane stability index $[13,24,28,29]$. Salinity also decreases photosynthesis through chlorophyll content reduction [30-32]. These biochemical and physiological damages are reflected in the poor growth of plants and reduce their overall productivity $[16,31,33]$.

Several options are available to solve salinity stress problems ranging from conventional plant breeding programs to the development of salt-tolerant crop plants by genetic engineering [34]. However, these conventional methods are time-consuming, laborintensive, rely on well-characterized germplasms, and may introduce undesirable traits along with the selected one $[30,35]$. The use of safe plant growth-promoting bacteria (PGPB) is an alternative option for drought and salinity stress mitigation [36-40]. Actinobacteria are considered a promising group of PGPB which offer an alternative environment-friendly way to mitigate the negative effects of salinity stress. Actinobacteria were reported to be potential plant growth promoters under normal and stress conditions. They act via mechanisms, such as phytohormone production, siderophore production, and phosphate solubilization [41-44]. Actinobacteria are also considered powerful biocontrol agents due to their antibiotic and lytic enzyme production to inhibit plant pathogens $[41,43,45,46]$. Streptomyces are one of the largest genera of Actinobacteria, with over 500 species [47]. These filamentous bacteria are well accepted as the most significant producer of bioactive compounds with extensive use in industrial applications [48-51]. Streptomyces are also widely used in agriculture as a supplement to enhance plant growth and inhibit plant pathogens-for instance, Streptomyces sp. KLBMP S0051 isolated from coastal salt marsh rhizosphere soils in China could produce indole-3-acetic acid (IAA) and promote seed germination of wheat variety Bainong AK58 and enhance seedling growth under salt stress [52]. Streptomyces spp. were also reported to promote growth in other plants, such as rice [53] and tomato [32,54]. Non-Streptomyces are also a good producer of bioactive compounds, with at least 2500 compounds reported [50]. However, research on nonStreptomyces actinobacteria to promote plant growth under abiotic stress is still limited. Micrococcus from forest soil in India showed plant growth-promoting traits in vitro and promoted cowpea growth [55]. Other non-Streptomyces genera with plant growth-promoting potential include Curtobacterium flaccumfaciens, which mitigated barley growth under salinity [56], Microbacterium sp. 3J1, which increased pepper growth under desiccation [57] and Arthrobacter strains MS-1 and MS-2, which promote the growth of alfalfa under non-saline and $100 \mathrm{mM} \mathrm{NaCl}$ [40].

The discovery of extremophilic actinobacteria is interesting because of their adaptations to extreme environments leading to the production of unique bioactive compounds [52]. Actinobacteria have been found in various marine environments, including mangroves [58-60] and deep-sea sediments [2,3,61]. Most plant growth-promoting actinobacteria are isolated from non-saline environments. Information on actinobacteria from marine habitats with plant growth promotion is still scarce but beginning to attract attention. For example, Nocardiopsis yanglingensis, Streptomyces jiujiangensis, S. psammoticus, and Pseudonocardia oroxyli from mangrove areas in Thailand can produce IAA, siderophore, and phosphate solubilization and promote growth of rice (Oryza sativa) seedlings under normal 
and salinity conditions up to $200 \mathrm{mM} \mathrm{NaCl}$ [60]. Similarly, mangrove-associated S. iranensis S2-SC16 with IAA, siderophore producing, and phosphate solubilization abilities promote Thai jasmine rice (O. sativa KDML105) seedlings. This isolate also showed potential as a biocontrol agent for rice Bakanae disease caused by Fusarium fujikuroi [53]. In addition, Saccharomonospora sp. from the marine soil of Morocco have shown in vitro that both solubilize phosphorous and produce IAA and siderophores [62]. Recently, coral-associated actinobacteria were reported to alleviate salt stress during the germination and photosynthesis of tobacco plants [63]. However, deep-sea actinobacteria have never been explored in terms of their benefits to plants. Members of the genus Dermacoccus had previously been isolated from the Challenger Deep of the Mariana Trench in the western Pacific Ocean $[3,7,64]$. We suggest that the ability of these deep-sea actinobacteria to survive under constant high-salinity and osmotic stress would be useful to help plant growth under salt stress. Hence, this research aims to determine the ability of two deep-sea actinobacteria, D. barathri MT2.1 $1^{\mathrm{T}}$ and D. profundi $\mathrm{MT} 2.2^{\mathrm{T}}$ which could tolerate up to $10 \% \mathrm{NaCl}$ to promote tomato growth (Solanum lycopersicum) under salinity stress. A type species of the genus, D. nishinomiyaensis DSM20448 ${ }^{\mathrm{T}}$ originated from a non-marine environment was included for comparison. The safety of these strains was also evaluated to ensure no negative effect on organisms in the environments according to the Environmental and human safety index (EHSI) [65].

\section{Results}

\subsection{Plant Growth-Promoting Properties In Vitro}

All 3 strains of actinobacteria could produce IAA in tryptophan-added culture broth in the range of $6.18-16.64 \mu \mathrm{g} \mathrm{mL} \mathrm{m}^{-1}$ (Table 1). D. nishinomiyaensis DSM20448 ${ }^{\mathrm{T}}$ produced the highest concentration of IAA of $16.64 \mu \mathrm{g} \mathrm{ml}^{-1}$ in the absence of salt stress. Under salt stress at $\mathrm{NaCl}$ concentrations of 150,300 , and $450 \mathrm{mM}$, IAA production of D. barathri MT2.1 $1^{\mathrm{T}}$ showed no significant difference between culture broth with or without $\mathrm{NaCl}$. Interestingly, D. nishinomiyaensis DSM20448 ${ }^{\mathrm{T}}$ and D. profundi MT2. $2^{\mathrm{T}}$ showed no significant difference in IAA production between $0 \mathrm{mM}$ and $150 \mathrm{mM} \mathrm{NaCl}$. However, IAA production of both strains increased at a higher $\mathrm{NaCl}$ level (300 and $450 \mathrm{mM} \mathrm{NaCl}$ ). All actinobacteria produced siderophores on CAS agar. In addition, all strains grew in King's B broth and produced a varying quantity of siderophores (Table 2). Hydroxamate type siderophore was produced in a significantly higher quantity than catecholate type. The highest production of hydroxamate and catecholate type siderophores was observed in D. barathri $\mathrm{MT} 2.1^{\mathrm{T}}$ at $240.00 \mu \mathrm{mol} \mathrm{mL}{ }^{-1}$ and D. nishinomiyaensis DSM20448 ${ }^{\mathrm{T}}$ at $22.63 \mu \mathrm{mol} \mathrm{mL}{ }^{-1}$, respectively. All strains produced a clear zone of tri-calcium phosphate solubilization on PVK agar, as shown in Table 2. Deep-sea Dermacoccus strains released higher phosphorus in the culture broth. The $\mathrm{pH}$ of the culture broth was reduced from 7 to below 5 (4.62-4.78). All Dermacoccus strains showed low growth on ACC containing minimal medium compared to minimal medium supplemented with ammonium sulfate (positive control). Therefore, they were not considered ACC deaminase-producing strains (Figure S2).

Table 1. Indole-3-acetic acid production $\left(\mu \mathrm{g} \mathrm{mL}^{-1}\right)$ of actinobacteria under various sodium chloride concentrations.

\begin{tabular}{|c|c|c|c|c|}
\hline Isolate & $\begin{array}{l}0 \mathrm{mM} \mathrm{NaCl}+ \\
\text { L-Tryptophan }\end{array}$ & $\begin{array}{c}150 \mathrm{mM} \mathrm{NaCl}+ \\
\text { L-Tryptophan }\end{array}$ & $\begin{array}{c}300 \mathrm{mM} \mathrm{NaCl}+ \\
\text { L-Tryptophan }\end{array}$ & $\begin{array}{c}450 \mathrm{mM} \mathrm{NaCl}+ \\
\text { L-Tryptophan }\end{array}$ \\
\hline DSM20448 ${ }^{\mathrm{T}}$ & $16.64^{\mathrm{a}} \pm 9.59$ & $9.39^{\mathrm{a}} \pm 5.47$ & $89.82^{b} \pm 12.15$ & $101.01^{b} \pm 8.61$ \\
\hline $\mathrm{MT} 2.1^{\mathrm{T}}$ & $6.18^{\mathrm{b}} \pm 0.82$ & $8.98^{b} \pm 0.82$ & $9.32^{b} \pm 1.22$ & $8.03^{\mathrm{b}} \pm 4.49$ \\
\hline $\mathrm{MT} 2.2^{\mathrm{T}}$ & $12.50^{\mathrm{a}, \mathrm{b}} \pm 10.61$ & $7.73^{a} \pm 0.73$ & $21.25^{\mathrm{b}} \pm 3.12$ & $19.76^{\mathrm{b}} \pm 4.48$ \\
\hline
\end{tabular}

Data represent the mean values of three replicates \pm SD. Different letters $(a, b)$ indicate a significant difference in IAA production in each $\mathrm{NaCl}$ concentration according to Duncan at $p<0.05$. 
Table 2. Siderophore production and phosphate solubilization of actinobacteria.

\begin{tabular}{|c|c|c|c|c|c|c|}
\hline \multirow[b]{2}{*}{ Isolate } & \multicolumn{3}{|c|}{ Siderophore Production } & \multicolumn{3}{|c|}{ Phosphate Solubilization } \\
\hline & $\begin{array}{c}\text { Yellow/Orange } \\
\text { Zone on CAS Agar }\end{array}$ & $\begin{array}{l}\text { Hydroxamate } \\
(\mu \mathrm{mol} \mathrm{mL}-1)\end{array}$ & $\begin{array}{l}\text { Catecholate } \\
(\mu \mathrm{mol} \mathrm{mL}-1)\end{array}$ & $\begin{array}{l}\text { Clear Zone } \\
\text { on PVK Agar }\end{array}$ & $\begin{array}{c}\text { P Released in PVK } \\
\text { Broth }\left(\mu \mathrm{g} \mathrm{mL}^{-1}\right)\end{array}$ & $\mathrm{pH}$ \\
\hline DSM20448 & + & $121.25^{\mathrm{a}} \pm 26.52$ & $22.63^{\mathrm{a}} \pm 3.20$ & + & $46.47^{\mathrm{a}} \pm 2.92$ & $4.78^{a} \pm 0.25$ \\
\hline $\mathrm{MT} 2.1^{\mathrm{T}}$ & + & $240.00^{b} \pm 21.21$ & $10.18^{\mathrm{c}} \pm 4.29$ & + & $169.27^{b} \pm 3.11$ & $4.62^{\mathrm{a}} \pm 0.04$ \\
\hline $\mathrm{MT} 2.2^{\mathrm{T}}$ & + & $129.38^{\mathrm{a}} \pm 27.40$ & $12.10^{\mathrm{c}} \pm 3.45$ & + & $165.47^{b} \pm 7.35$ & $4.66^{\mathrm{a}} \pm 0.06$ \\
\hline
\end{tabular}

+: Positive results. Data represent the mean values of three replicates \pm SD. Different letters $(a, b)$ indicate a significant difference in siderophore production and phosphate solubilization among strains according to Duncan at $p<0.05$.

\subsection{Enhancement of Tomato Seedlings Growth without Salt Stress}

Dermacoccus strains were applied to tomato seedlings to evaluate their growth-promoting effects (Figure 1a-d). The inoculation of Dermacoccus strains increased the growth of tomato seedlings compared to non-inoculated control in terms of dry weight (Figure 1d) and root length (Figure 1b). D. barathri MT2.1 $1^{\mathrm{T}}$ significantly promoted the root length in comparison to the non-inoculated control or other Dermacoccus strains $(p<0.05)$.
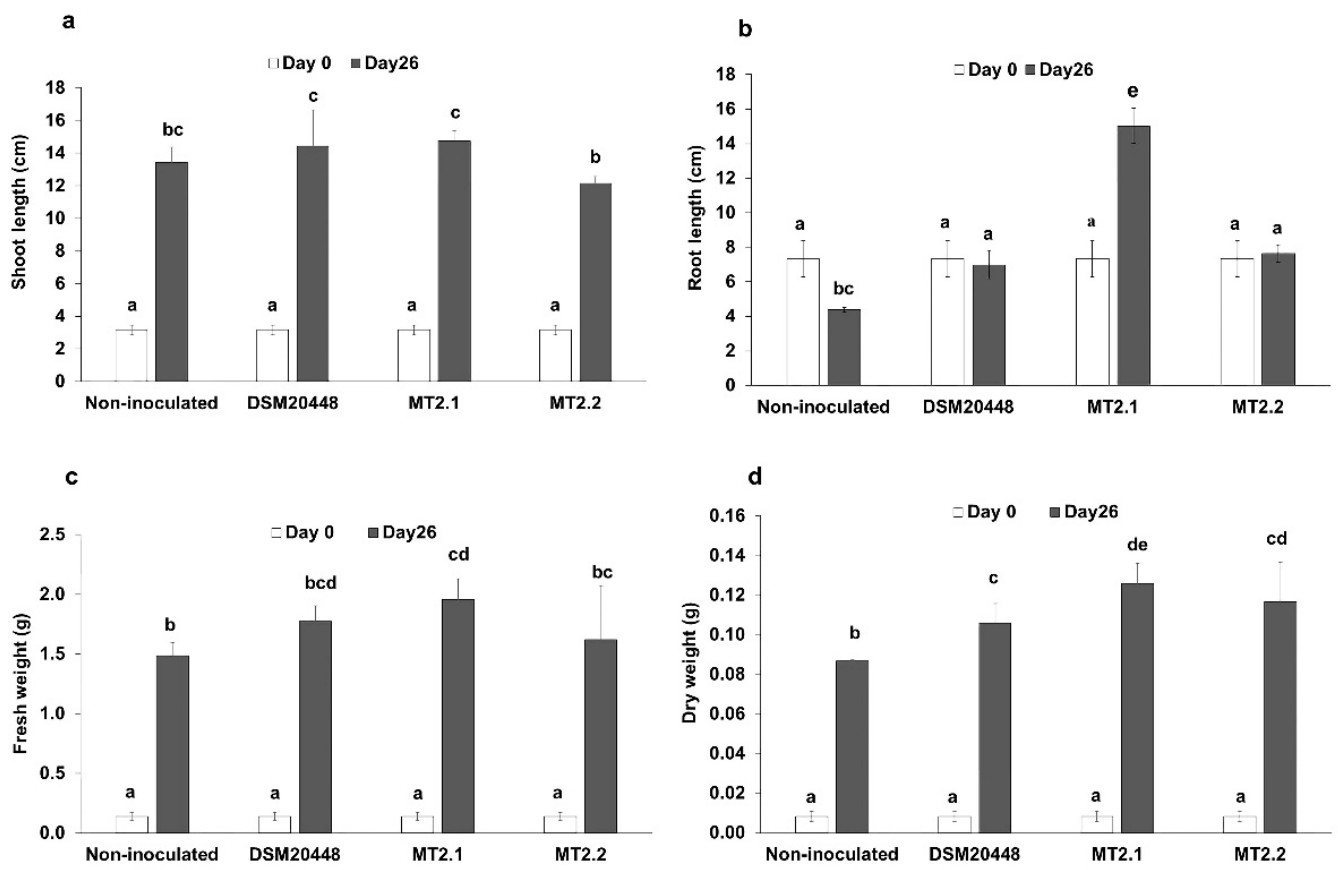

Figure 1. Deep-sea actinobacteria promote tomato seedlings' growth without salt stress. (a) shoot length; (b) root length; (c) fresh weight; (d) dry weight. Data represent the mean values of three replicates. Different letters (a,b,c,e,de,cd,bc,bcd) indicate a significant difference according to Duncan at $p<0.05$.

\subsection{Enhancement of Tomato Seedlings Growth under Salt Stress}

The effects of Dermacoccus strains on tomato seedlings under salinity stress at $150 \mathrm{mM}$ $\mathrm{NaCl}$ are shown in Figures 2 and 3. In general, tomatoes inoculated with Dermacoccus strains showed better growth than non-inoculated seedlings. At the end of the experiment (14 days), tomato inoculated with $D$. barathri MT2.1 ${ }^{\mathrm{T}}$ showed shoot length (Figure 2a), root length (Figure $2 b$ ), fresh weight (Figure 2c), and dry weight (Figure 2d) significantly higher than non-inoculated tomato under stress. Moreover, tomato inoculated with D. nishinomiyaensis DSM20448 ${ }^{\mathrm{T}}$ showed no significant differences in shoot length, root length, dry weight, and fresh weight compared with non-inoculated tomato seedlings under salt stress (Figure 2a-d). 

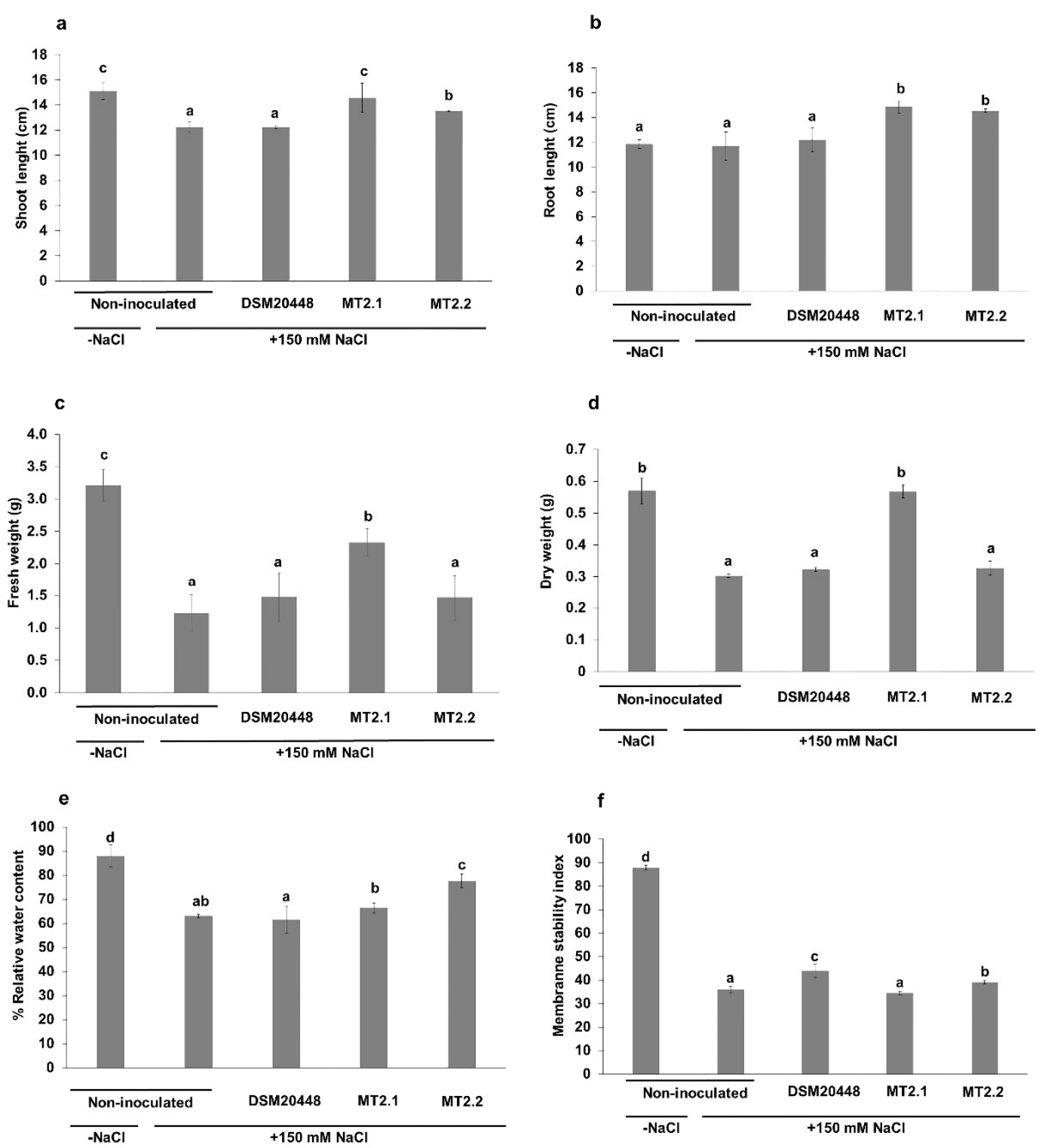

Figure 2. Effect of deep-sea actinobacteria on physiological properties of tomato seedlings under salinity stress (150 mM $\mathrm{NaCl}$ ). (a) shoot length; (b) root length; (c) fresh weight; (d) dry weight; (e) percentage of relative water content (\% RWC); (f) membrane stability index (MSI). Data represent the mean values of three replicates $\pm \mathrm{SD}$. Different letters (a,b,c,d) indicate a significant difference according to Duncan at $p<0.05$. $-\mathrm{NaCl}$ means non-stressed condition; $+150 \mathrm{mM} \mathrm{NaCl}$ means salinity stress condition.

Furthermore, when comparing tomato inoculated with deep-sea strains (D. barathri MT2. $1^{\mathrm{T}}$ and D. profundi $\mathrm{MT} 2.2^{\mathrm{T}}$ ) and a terrestrial strain (D. nishinomiyaensis DSM20448 ${ }^{\mathrm{T}}$ ), deep-sea strains showed better shoot and root length in tomato seedling under salt stress. In particular, D. barathri MT2. $1^{\mathrm{T}}$ showed the highest values of shoot length, root length, fresh weight, and dry weight (Figure 2a-d). In addition, similar shoot length and dry weight were observed between tomato inoculated with D. barathri $\mathrm{MT} 2 \cdot 1^{\mathrm{T}}$ and control tomato without salt stress (non-inoculated) (Figure 2a,d).

The highest percentage of RWC was observed in non-stressed tomato, while tomatoes inoculated with deep-sea strains of D. barathri MT2.1 $1^{\mathrm{T}}(66.39 \%)$ and D. profundi MT2.2 ${ }^{\mathrm{T}}$ (77.62\%) showed a higher RWC than inoculated tomato seedlings with D. nishinomiyaensis DSM20448 ${ }^{\mathrm{T}}(61.59 \%)$ under stress conditions (Figure 2e). However, tomato-inoculated with D. barathri MT2. $1^{\mathrm{T}}$ and D. nishinomiyaensis DSM $20448^{\mathrm{T}}$ showed no significant difference in RWC compared with non-inoculated tomato (62.99\%) under salt stress (Figure 2e). MSI in 
all tomatoes inoculated with actinobacteria under salinity stress was significantly higher than in non-inoculated tomatoes with stress except for D. barathri MT2.1 $1^{\mathrm{T}}$ (Figure 2f).
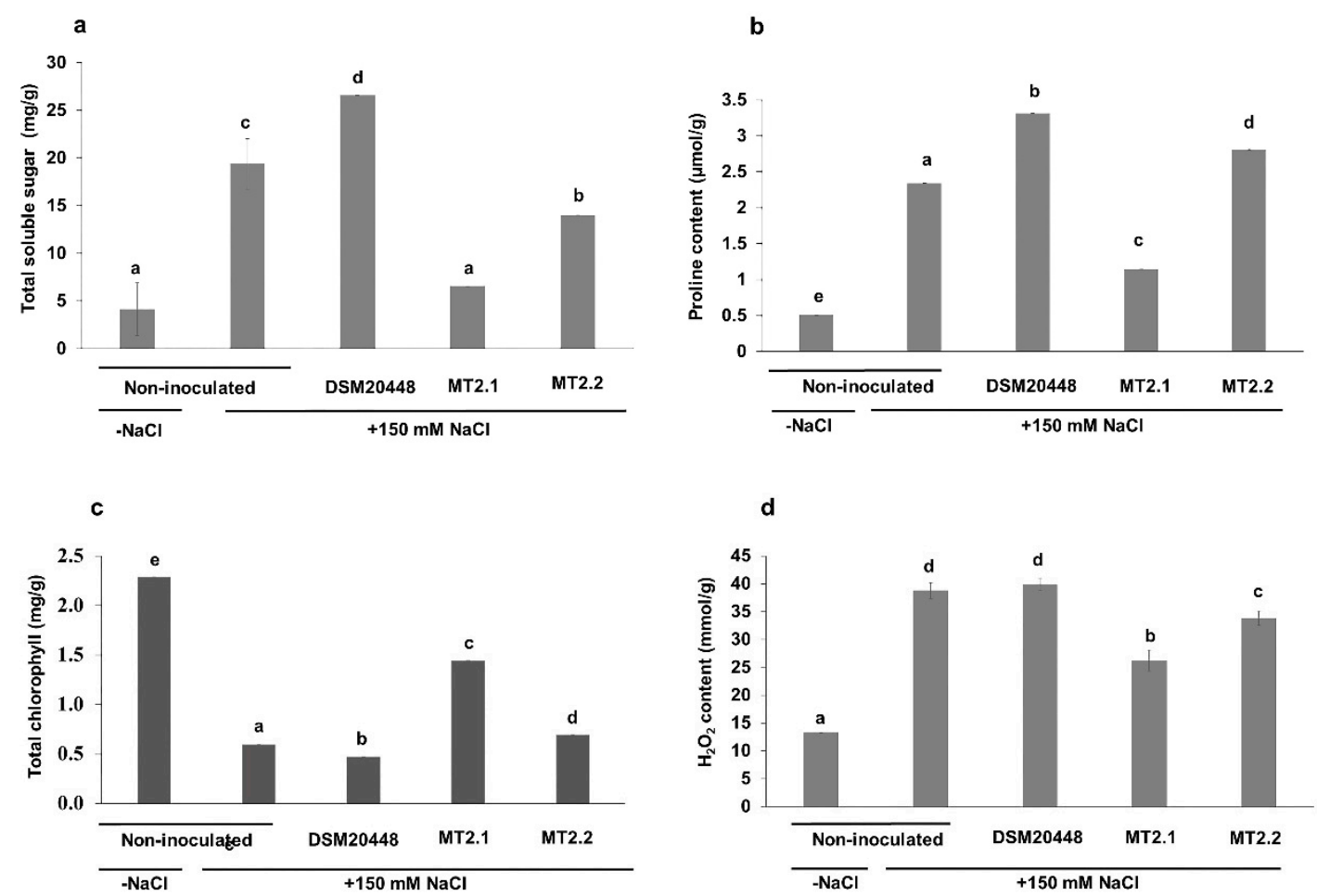

Figure 3. Effect of deep-sea actinobacteria on biochemical properties of tomato seedlings under salinity stress (150 mM $\mathrm{NaCl}$ ). (a) total soluble sugar; (b) proline content; (c) total chlorophyll; (d) content of hydrogen peroxide. Data represent the mean values of three replicates $\pm S D$. Different letters $(a, b, c, d)$ indicate a significant difference according to Duncan at $p<0.05$. $-\mathrm{NaCl}$ means non-stressed condition; $+150 \mathrm{mM} \mathrm{NaCl}$ means salinity stress condition.

The effect of salt stress on the morphology of tomato seedlings was also recorded (Figure S1). At the end of the experiment, non-inoculated tomato seedlings under stress conditions showed curly and wilting leaves and faded leaf color (Figure S1b). However, tomato seedlings inoculated with deep-sea Dermacoccus strains showed better overall growth (Figure S1c-e). Leaves on the upper part of tomato seedling-inoculated with deepsea Dermacoccus strains were still healthy compared to non-inoculated with stress tomato seedlings. Moreover, the root architecture of inoculated seedlings was improved as observed from an increased length of the main root and the number of lateral root branching.

\section{Biochemical Analysis}

The biochemical response of tomato seedlings with inoculated actinobacteria in mitigating salinity stress was determined in proline content, TSS, hydrogen peroxide content, and chlorophyll content (Figure 3a-d). For TSS and proline contents, the inoculation of tomato seedlings with actinobacteria resulted in a varied response in a species-dependent manner. For example, tomato seedlings inoculated with D. nishinomiyaensis DSM20448 ${ }^{\mathrm{T}}$ showed higher TSS and proline content than the non-inoculated tomato under salt stress. However, there is a pronounced and significant decrease of TSS in tomato seedlings inoculated with both the deep-sea strains and of proline with $D$. barathri MT2.1 $1^{\mathrm{T}}$ than non-inoculated tomato under salt stress (Figure $3 a, b$ ). Interestingly, tomatoes inoculated with D. barathri MT2.1 $1^{\mathrm{T}}$ showed a similar TSS level to the non-inoculated tomato plants under non-stressed conditions (Figure 3a). Higher proline accumulation was observed in all stressed tomato plants with and without inoculated actinobacteria (Figure $3 b$ ). Total chlorophyll content in deep-sea Dermacoccus inoculated tomato seedlings was significantly higher than non-inoculated tomatoes under stressing conditions. Plants inoculated with 
D. nishinomiyaensis DSM20448 ${ }^{\mathrm{T}}$ presented the lowest chlorophyll content, whereas the highest content was observed in non-inoculated plants in the absence of salt stress (Figure 3c). The hydrogen peroxide content $\left(\mathrm{H}_{2} \mathrm{O}_{2}\right)$ in tomato seedlings inoculated with the deep-sea Dermacoccus was lower than in the non-inoculated tomato and tomato inoculated with D. nishinomiyaensis DSM20448 ${ }^{\mathrm{T}}$, both of them under salty stress (Figure $3 \mathrm{~d}$ ). In addition, non-inoculated tomato plants in non-stressing conditions showed the lowest hydrogen peroxide value compared to tomato plants under salt stress regardless of the addition of actinobacteria (Figure 3d).

\subsection{Biosafety Tests for Actinobacteria}

\subsubsection{Pathogenicity Bioassay Based on Caenorhabditis elegans}

Pathogenicity bioassay was performed to evaluate the effect of actinobacteria on the number of adults, juveniles, eggs, and dead C. elegans (Figure $4 a-d$ ). When fed with P. aeruginosa PA14 (as pathogenic strain), the lowest number of adults, juveniles, and eggs were found, and all nematodes died after $96 \mathrm{~h}$. The highest number of adults, juveniles, and eggs were found when fed with E. coli OP50 (as a non-pathogenic strain) at $144 \mathrm{~h}$. Nematodes fed with $D$. barathri MT2.1 $1^{\mathrm{T}}$ showed a $51 \%$ reduction in the number of adults and $31 \%$ of juveniles compared to control (E. coli OP50). For D. nishinomiyaensis DSM20448 ${ }^{\mathrm{T}}$, the number of adults and juveniles decreased by 59\% and $92 \%$. The highest reduction (96\%) in the number of adults and juveniles was observed in nematodes fed with $D$. profundi MT2. $2^{\mathrm{T}}$. The number of eggs at $144 \mathrm{~h}$ was similar between E. coli OP50 (123 eggs) and D. barathri MT2.1 ${ }^{\mathrm{T}}$ (120 eggs). However, nematodes fed with D. nishinomiyaensis DSM20448 ${ }^{\mathrm{T}}$ did not hatch an egg at $144 \mathrm{~h}$, and D. profundi $\mathrm{MT} 2.2^{\mathrm{T}}$ showed the lowest number of eggs (12 eggs). For the percentage of death at $144 \mathrm{~h}, P$. aeruginosa PA14 showed the highest death $(100 \%)$, followed by D. profundi MT2.2 $2^{\mathrm{T}}(23 \%)$, D. nishinomiyaensis DSM $20448^{\mathrm{T}}(11 \%)$, D. barathri MT2.1 ${ }^{\mathrm{T}}(1.8 \%)$, and E. coli OP50 (1.6\%) (Figure $\left.4 \mathrm{~d}\right)$.
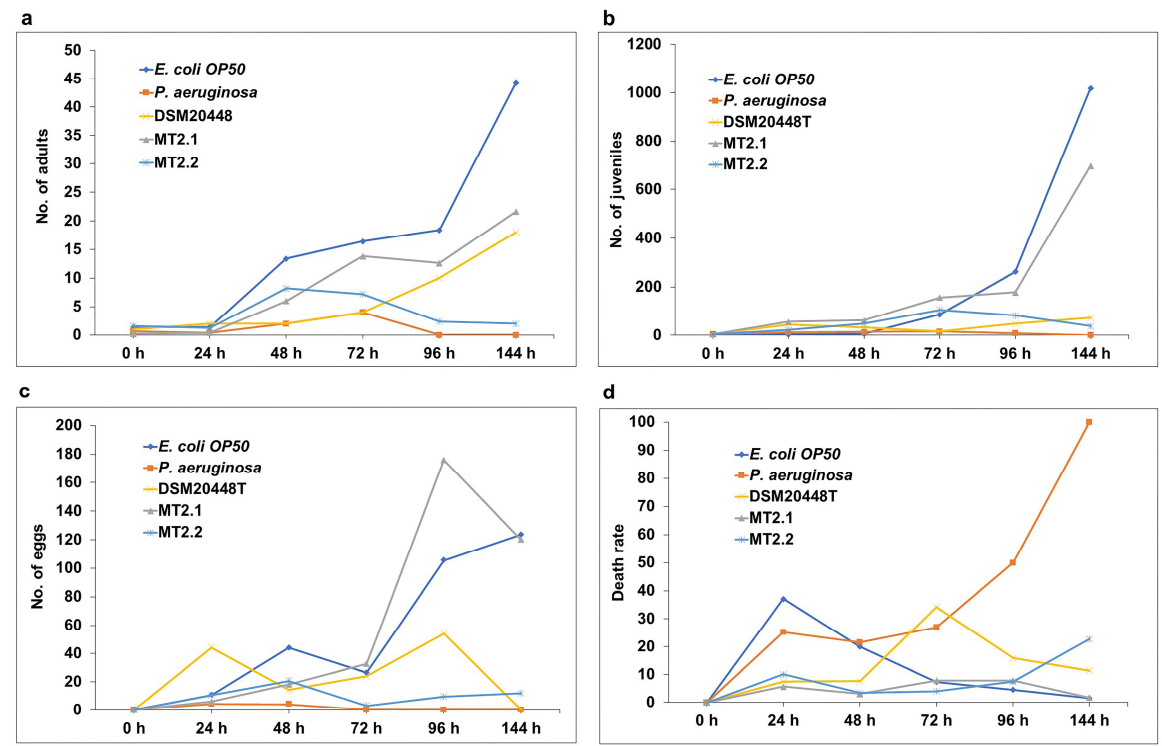

Figure 4. Pathogenicity bioassay based on Caenorhabditis elegans. Time course of changes in the number of (a) adults; (b) juveniles; (c) eggs; (d) death rate. Data represent the mean values of three replicates \pm SD. Different letters indicate a significant difference according to Duncan at $p<0.05$.

\subsubsection{Escherichia coli MC4100 Sensitivity}

Some actinobacteria can produce and release secondary metabolites to the environment affecting the microbial community. Therefore, we investigated the effects of the supernatant of actinobacteria on E. coli MC4100 cells (Figure 5). Survival rate (\%) of E. coli MC4100 cells when exposed to cell-free supernatant from P. putida KT2440, D. barathri MT2.1 ${ }^{\mathrm{T}}$, and D. profundi MT2.2 ${ }^{\mathrm{T}}$ were $91.9 \%, 91.9 \%$, and $86.5 \%$, respectively. However, 
more than $40 \%$ reduction in E. coli MC4100 cells was found when exposed to supernatants of P. aeruginosa PA14 (41.9\%) and D. nishinomiyaensis DSM20448 ${ }^{\mathrm{T}}(61.5 \%)$.

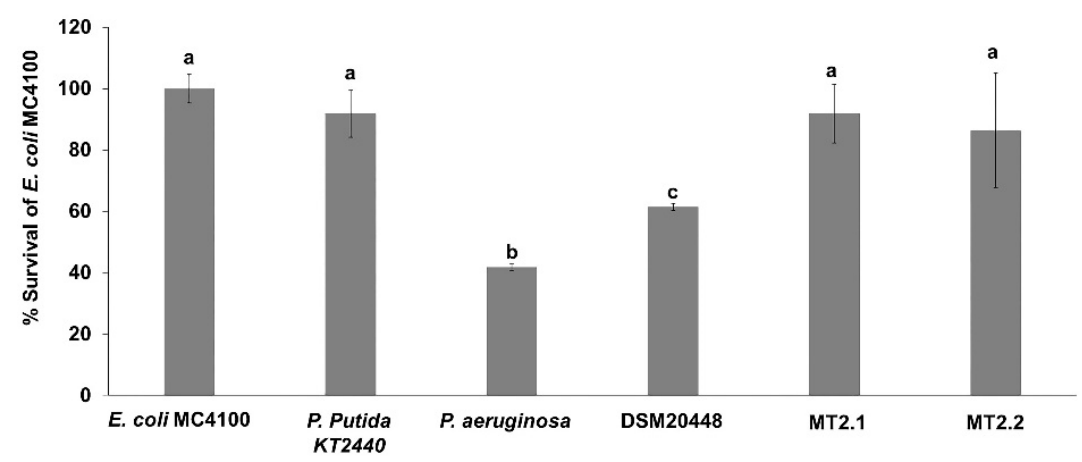

Figure 5. Escherichia coli MC4100 sensitivity. Data represent the mean values of three replicates \pm SD. Different letters $(\mathrm{a}, \mathrm{b}, \mathrm{c})$ indicate a significant difference according to Duncan at $p<0.05$.

\subsubsection{Ecotoxicity Test in Earthworms (Eisenia foetida)}

The effects of actinobacteria on annelids in soil were investigated using earthworms (E. foetida). The development of earthworms was determined by measuring the weight, length, and number of ootheca (Figure 6). At day 21, a similar pattern of increasing weight in earthworms fed with only chickpea (positive control, $75 \%$ ) or chickpea supplemented with different bacteria: P. putida KT2440 (safe strain; 66\%), B. cepacia CC-A174 (pathogenic strain; 75\%), D. nishinomiyaensis DSM20448 ${ }^{\mathrm{T}}(75 \%)$ and D. barathri MT2.1 ${ }^{\mathrm{T}}$ $(77 \%)$ was found. A similar increasing trend of earthworm length was also observed when fed with bacteria-supplemented chickpea (46-55\%) compared with non-supplemented chickpea. The addition of $2 \% \mathrm{NaCl}$ resulted in the lowest value for both the weight and length of earthworms. For the number of eggs, the addition of P. putida KT2440 (12.5 eggs/earthworm) showed the highest number of ootheca, followed by D. nishinomiyaensis DSM $20448^{\mathrm{T}}$ (10.4 eggs/earthworm), chickpea (10.3 eggs/earthworm), D. barathri $\mathrm{MT} 2.1^{\mathrm{T}}$ (10 eggs/earthworm) and B. cepacia CC-A174 (9.6 eggs/earthworm). The lowest number of ootheca was observed in chickpea supplemented with $2 \%(w / w)$ $\mathrm{NaCl}$ (4.8 eggs/earthworm) (data not shown).

\subsubsection{Daphnia magna Toxicity Bioassay}

Daphnia magna was used to study the impact of microbial metabolites on the aquatic ecosystem. TSB was used for suspension preparation and standard freshwater as control. Standard freshwater showed no effect on D. magna. The addition of spent media from B. cepacia CC-A174 had the most adverse effect on D. magna survival as all Daphnia were dead at the lowest concentration tested (3.125\%). The EC50 value of D. barathri MT2.1 ${ }^{\mathrm{T}}$ was $6.25 \%$ at $24 \mathrm{~h}$. However, the addition of spent media at this concentration from the culture of both D. nishinomiyaensis DSM20448 ${ }^{\mathrm{T}}$ and P. putida KT2440 had no adverse effect on D. magna (Data not shown). 
a

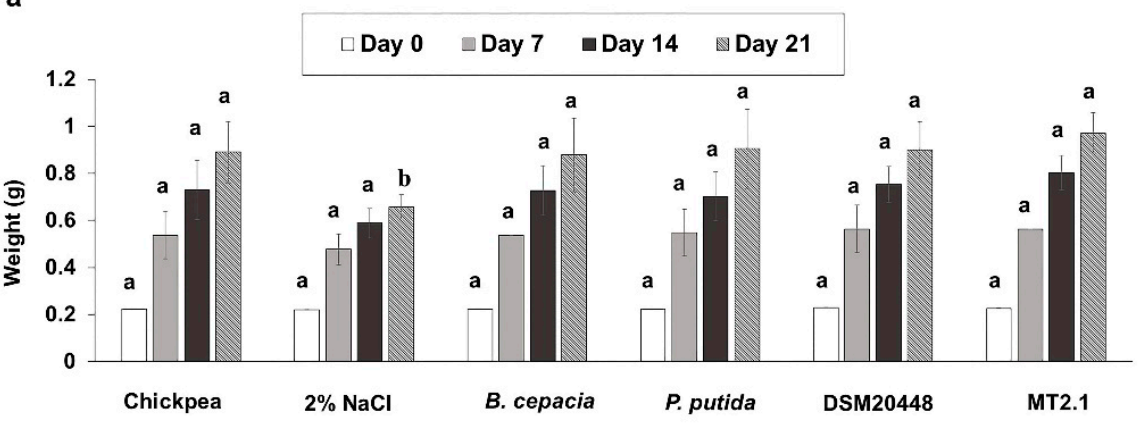

b

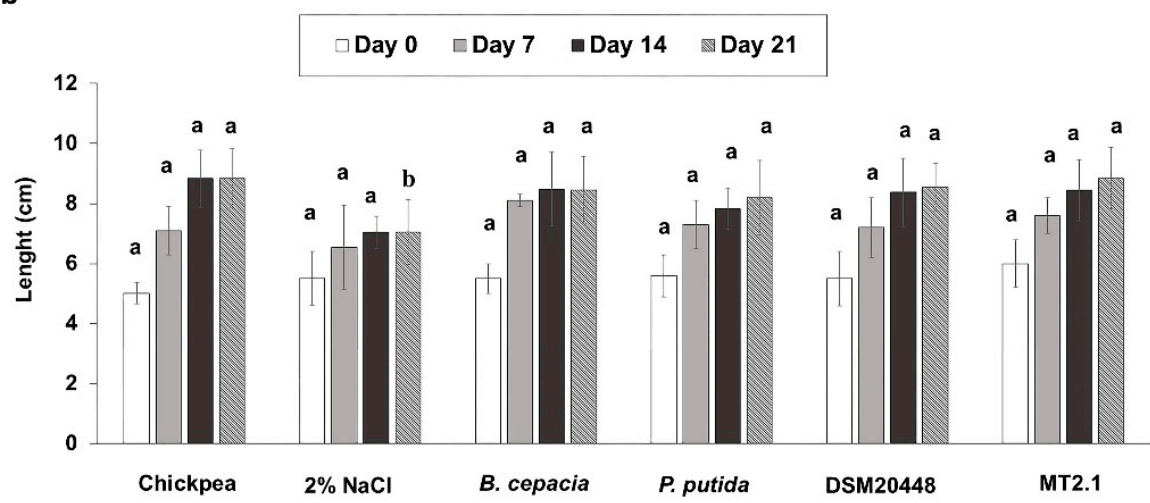

Figure 6. Ecotoxicity tests in earthworms (Eisenia foetida). Time course of changes in (a) weight; (b) length of Eisenia foetida after incubation with B. cepacia CC-A174 or P. putida KT2440 or D. nishinomiyaensis DSM20448 ${ }^{\mathrm{T}}$ or D. barathri MT2.1 $1^{\mathrm{T}}$. Data represent the mean values of three replicates \pm SD. Different letters $(a, b)$ indicate a significant difference in weight and length of earthworms at different cultivation days according to Duncan at $p<0.05$.

\section{Discussion}

\subsection{Plant Growth-Promoting Properties In Vitro}

Indole-3-acetic acid (IAA) is a major auxin-type phytohormone that helps the plant grow and develop. It also contributes to the growth of young seedlings [26], embryo and fruit development, and especially root hair formation [66]. Many actinobacteria were reported to produce IAA, such as Arthrobacter sp., Nocardioides sp. [67], Pseudonocardia sp. [68], and Streptomyces spp. [68,69]. Marine actinobacteria were also able to produce IAA, e.g., Streptomyces spp. isolated from the Egyptian coastal area produced IAA in the range of 4.12-49.7 $\mu \mathrm{g} \mathrm{mL} \mathrm{m}^{-1}$ [70]. Saccharomonospora sp. LNS-1 isolated from the largest lagoon in Morocco produces $49.46 \mu \mathrm{g} \mathrm{mL}^{-1}$ IAA in vitro [62]. In the present study, deep-sea D. barathri MT2.1 $1^{\mathrm{T}}$ and D. profundi $\mathrm{MT} 2 \cdot 2^{\mathrm{T}}$ showed lower IAA production than a terrestrial strain of D. nishinomiyaensis DSM20448 ${ }^{\mathrm{T}}$ in tryptophan-added culture broth without $\mathrm{NaCl}$. All strains still produced IAA under increased $\mathrm{NaCl}$ concentration up to $450 \mathrm{mM} \mathrm{NaCl}$. An increasing $\mathrm{NaCl}$ concentration did not affect the IAA production of D. barathri MT2.1 ${ }^{\mathrm{T}}$. However, $\mathrm{NaCl}$ concentration seems to have both negative and positive effects on the IAA production of D. nishinomiyaensis DSM20448 ${ }^{\mathrm{T}}$ and D. profundi MT2.2 ${ }^{\mathrm{T}}$. At low $\mathrm{NaCl}$ concentration $(150 \mathrm{mM} \mathrm{NaCl})$, IAA production was reduced in both $D$. nishinomiyaensis DSM20448 ${ }^{\mathrm{T}}$ and D. profundi MT2.2 $2^{\mathrm{T}}$ (Table 1). On the contrary, IAA production was increased in both strains at higher $\mathrm{NaCl}$ concentrations (300 and $450 \mathrm{mM} \mathrm{NaCl}$ ). A similar observation was found in Streptomyces isolate $C$ isolated from Iranian wheat field; that is, the IAA production increased $\left(2.4-4.7 \mu \mathrm{g} \mathrm{mL}^{-1}\right)$ with an increasing $\mathrm{NaCl}$ concentration from 100-300 $\mathrm{mM}$ [71]. The production of IAA from marine actinobacteria is rarely reported. The only available information was from mangrove actinobacteria $[53,60]$. No attempt had been previously performed to investigate plant growth-promoting properties in deep-sea 
actinobacteria, including IAA production. However, the mechanism on how $\mathrm{NaCl}$ inserts a positive effect on IAA production remains unclear and needs additional research.

Siderophores are ferric iron $\left(\mathrm{Fe}^{3+}\right)$ specific chelators, facilitating iron uptake under limiting iron [72,73]. Siderophores are produced by some microorganisms, including actinobacteria. Siderophores are known to promote plant growth via both direct (iron uptake) and indirect mechanisms (pathogen inhibition) [71,74]. Siderophores are classified into hydroxamate, catecholate, and carboxylate types. Interestingly, Dermacoccus spp. produced higher hydroxamate than catecholate type siderophore in King's B broth. Phosphorus is one of the major macronutrients required for plant growth. Phosphorus in the soil is found in an insoluble form that cannot be used by plants. However, some microorganisms can solubilize phosphate and release phosphorus into the soil. In the present study, all strains solubilized tri-calcium phosphate in the culture broth. Liquid media was more suitable for determining phosphate solubilization by bacteria [75]. A drop in $\mathrm{pH}$ of culture broth to $4.62-4.78$ was also observed. A reduction in the $\mathrm{pH}$ of the media from organic acid production is a known primary mechanism of phosphate solubilization in microorganisms, including actinobacteria $[68,76]$. Deep-sea Dermacoccus strain released phosphorus almost 4 times higher than their terrestrial neighbor, D. nishinomiyaensis DSM20448 ${ }^{\mathrm{T}}$. High phosphate solubilizing activity was also reported from members of genera Streptomyces, Microbacterium, Angusibacter, Kocuria, Isoptericola, and Agromyces isolated from sediment in Chorao Island, India [77].

The enzymatic activity of ACC deaminase results in the degradation of ACC, which is the precursor of ethylene in all higher plants [12]. Ethylene level in plants is increased under salinity stress, leading to series of abnormal morphologies, such as leaf yellowing, the senescence of various organs, the abscission of leaves, petals, and flowers, and premature death [12,78]. A lower level of ACC reduced ethylene synthesis resulting in lower stress levels in plants. However, all Dermacoccus strains showed no ACC deaminase activity. This observation suggested that these Dermacoccus strains used alternative mechanisms to alleviate salinity stress in tomato seedlings.

\subsection{Promotion of Tomato Growth Using Dermacoccus Strains}

Inoculation of Dermacoccus strains positively affected the growth of tomato seedlings under non-stressed conditions as seen from the dry weight and root length compared to non-inoculated seedlings. Although studies of marine actinobacteria to enhance plant growth are still limited, actinobacteria from other saline habitats were used as plant growth promotors. Only actinobacteria from mangrove in Thailand, which belong to the genera Nocardiopsis, Pseudonocardia, and Streptomyces were reported to promote the growth of rice seedlings without salt stress and up to $200 \mathrm{mM} \mathrm{NaCl}$ [60]. Similarly, Streptomyces sp. KLBMP S0051 from Chinese coastal salt marsh rhizosphere soils, promoted the growth of wheat seedlings with and without $\mathrm{NaCl}$ [52]. Recently, Glutamicibacter halophytocola KLBMP 5180 isolated from the root of Chinese coastal halophyte, increased the growth of tomato seedlings under the non-saline condition in terms of fresh weight, height, root length, number of fibrous roots, and total chlorophyll content [79]. It is evident from ours results that deep-sea Dermacoccus have no negative effect on tomato growth and were selected to test their ability to promote the growth of tomato seedlings under salinity stress.

Although the ecological roles of marine actinobacteria remain largely unknown and they need to be determined, actinobacteria from the deep-sea environments have to constantly tolerate salinity, high pressure, low temperature, and frequently low nutrient availability. It is not surprising that they have to evolve unique properties to survive under such extreme environments. The Mariana Trench, the deepest location on earth, is remarkably high in hydrostatic pressure and salinity [80]. Recently, genes related to stress response, especially those related to osmotic stress response, e.g., synthesis and accumulation of compatible solutes, and oxidative stress response, e.g., ROS scavenging enzymes, which may play an essential role in the evolution of marine actinobacteria to tolerate salinity stress were reported for D. abyssi MT1.1 ${ }^{\mathrm{T}}$ [81]. From this evidence, we 
designed the experiment to test the ability of two other deep-sea Dermacoccus from the Mariana Trench to mitigate salt stress on tomato growth.

Salt stress is one of the most important abiotic stress limiting agricultural productivity. Soil salinity causes an alteration in biochemical and physiological properties, such as ion toxicity, osmotic stress, mineral uptake, nutrient deficiency, and photosynthetic activity [14,71]. From our results, salinity stress at $150 \mathrm{mM} \mathrm{NaCl}$ clearly asserted a negative effect on tomato morphology (Figure S1b) as observed in the number of wither leaves, the reduced main root length, and the number of lateral root branching. A similar observation in tomato (Solanum lycopersicum L.) was reported by Karni et al. [82] who found that $100 \mathrm{mM} \mathrm{NaCl}$ reduced the length of the main root. However, an increase in the number of branching roots was also recorded. This inconsistency is not surprising because the lateral root formation can be less affected or might even be stimulated by the salinity stress [83].

In the present study, we evaluated the effects of deep-sea actinobacteria on tomato seedlings under salt stress $(150 \mathrm{mM} \mathrm{NaCl})$. As observed, deep-sea actinobacteria (D. barathri $\mathrm{MT} 2.1^{\mathrm{T}}$ and D. profundi $\mathrm{MT} 2.2^{\mathrm{T}}$ ) were able to improve tomato seedling appearance as tomatoes inoculated with these two strains showed higher shoot and root length than non-inoculated tomato plants under salt stress (Figure 2a,b). Similar results were stated by Tank et al. [84] who reported the ability of rhizobacteria isolated from tomato fields to increase tomato growth (shoot and root length, numbers of leaves, and lateral roots) under $2 \% \mathrm{NaCl}$. In addition, inoculation of tomatoes with $D$. barathri MT2.1 ${ }^{\mathrm{T}}$ resulted in a higher shoot length, root length, fresh and dry weight than non-inoculated tomato plants under stress conditions. Palaniyandi et al. [31] isolated Streptomyces PGPA39 from agricultural soils, which alleviated salt stress $(180 \mathrm{mM} \mathrm{NaCl})$ in Micro-Tom tomato. This strain improved shoot length, root length, and dry biomass of tomato due to IAA production. Production of IAA by plant growth-promoting rhizobacteria has been reported that helps in root formation and root architecture modification, resulting in an increased root area and nutrient uptake efficiency of plants under salinity stress [15]. It is interesting to note that tomato inoculated with a terrestrial strain of D. nishinomiyaensis DSM20448 ${ }^{\mathrm{T}}$ showed no significant difference in growth performance in terms of shoot length, root length, fresh and dry weight compared with non-inoculated tomato under stress. Our results show that salinity stress negatively affected tomato growth in terms of shoot length, fresh weight, and dry weight. In addition, we found that the inoculation of deep-sea D. barathri MT2.1 $1^{\mathrm{T}}$ clearly mitigated salt stress in tomatoes as these parameters were either equivalent or even higher than in non-inoculated tomatoes without stress (Figure 2a,b,d).

The RWC and MSI are two important physiological indices for evaluating salt stress effects $[28,85]$. Salt stress induces an excess of $\mathrm{Na}^{+}$and $\mathrm{Cl}^{-}$ions; consequently, it reduces plant water uptake ability, reflecting in a lower RWC $[28,84]$. Therefore, RWC is an indicator of plant water status used as an index for dehydration tolerance [86]. Our results show that RWC values of $D$. profundi MT2. $2^{\mathrm{T}}$-inoculated tomato seedlings were higher than in non-inoculated control under salt stress. These results suggested that this strain could help to maintain water status in tomatoes under salt stress. Similar results were reported in barley-inoculated with rare actinobacteria, Curtobacterium flaccumfaciens E108, and Ensifer garamanticus E110 under $4.4 \% \mathrm{NaCl}$ concentration [56]. In contrast, tomato inoculated with neither D. barathri MT2.1 $1^{\mathrm{T}}$ or D. nishinomiyaensis DSM $20448^{\mathrm{T}}$ showed no significant difference with non-inoculated tomato plants under salt stress. Under salinity stress, the accumulation of salts in plant cells would damage the cell membrane integrity resulting in lower MSI [28]. Tomatoes inoculated with D. nishinomiyaensis DSM20448 ${ }^{\mathrm{T}}$ and D. profundi MT2.2 ${ }^{\mathrm{T}}$ showed higher MSI than non-inoculated tomatoes under $150 \mathrm{mM}$ $\mathrm{NaCl}$. Similar results were found in alfalfa plants inoculated with either Hartmannibacter diazotrophicus or Pseudomonas sp., which showed higher MSI than non-inoculated plants after 2 weeks at $10 \mathrm{dS} \mathrm{m}^{-1}$ and $20 \mathrm{dS} \mathrm{m}^{-1}$ salinity. The higher value of MSI indicates lower absorption of $\mathrm{Na}^{+}$ions into the plant cells [13]. D. barathri MT2.1 ${ }^{\mathrm{T}}$ inoculated tomato showed lower MSI that was not significantly different from the non-inoculated tomato seedlings control. Although inoculation of deep-sea D. barathri MT2.1 ${ }^{\mathrm{T}}$ could not restore 
RWC and MSI value to normal status as in non-inoculated tomato plants without stress, salinity stress did not affect the overall growth of inoculated tomato as observed in shoot length, root length, and dry weight (Figure 2a,b,d).

Soluble sugars play an important role in maintaining the overall structure and development of plants to mitigate the negative effects of salt $[87,88]$. For example, sugars act as regulators of metabolism, photosynthesis, osmotic homeostasis, protein synthesis, and gene expression during various abiotic stress [88]. However, in the present study, the accumulation of TSS showed no positive effect on protecting tomato seedlings from salt stress. The concentration of TSS in tomato seedlings inoculated with D. barathri MT2.1 ${ }^{\mathrm{T}}$ and D. profundi MT2.2 $2^{\mathrm{T}}$ was lower than that in non-inoculated tomato plants under stress conditions. Although the inoculation of tomato seedlings with D. nishinomiyaensis DSM20448 ${ }^{\mathrm{T}}$ showed the highest TSS, no improvement in the overall growth performance as exemplified in the recorded dry weight compared with tomato seedlings inoculated with deep-sea Dermacoccus was found (Figure $2 \mathrm{~d}$ ). These results suggested that both D. barathri MT2.1 ${ }^{\mathrm{T}}$ and D. profundi MT2.2 ${ }^{\mathrm{T}}$ did not use TSS to mitigate salinity stress. Hu et al. [89] reported that the addition of a low concentration of exogenous glucose $(0.1,0.5 \mathrm{mM})$ under salt stress (200 mM NaCl) enhanced seed germination of wheat, increased chlorophyll content, dry weight, and proline accumulation, maintained ionic homeostasis, prevented water loss and stimulated antioxidant enzyme activity. Similarly, the addition of a lower concentration of exogenous glucose and sucrose promoted the growth of triticale $(\times$ Triticosecale Wittmack) seedlings under salt stress $(100 \mathrm{mM} \mathrm{NaCl})$ [90].

Proline is a compatible solute accumulated by plants under various stress conditions, such as salinity, water shortage, and extreme temperature [91,92]. Under salinity, plants accumulate proline in the cytoplasm to adjust osmotic pressure against salt-stress-induced water loss from cells [93]. In the present study, tomato inoculated with D. nishinomiyaensis DSM20448 ${ }^{\mathrm{T}}$ and D. profundi MT2.2 $2^{\mathrm{T}}$ increased proline content in tomato leaves. However, only tomato seedlings inoculated with D. barathri MT2.1 $1^{\mathrm{T}}$ showed better growth in all parameters (shoot length, root length, fresh and dry weight) than non-inoculated tomatoes under stress. Previously, studies on mungbean using Bacillus cereus Pb25 showed an increase in proline content in inoculated plants under salinity $\left(\mathrm{NaCl} 9 \mathrm{dS} \mathrm{m}^{-1}\right)$ and increased fresh and dry root and shoot weight and yield compared to control [93]. On the contrary, proline content in tomatoes inoculated with D. barathri $\mathrm{MT} 2.1^{\mathrm{T}}$ was reduced. Although the inoculation of D. barathri $\mathrm{MT} 2.1^{\mathrm{T}}$ resulted in lower proline content in tomato leaves, the tomato seedlings showed better overall growth performance than non-inoculated tomatoes under stress and other treatments. In agreement with our results, Palaniyandi et al. [31] reported that tomatoes inoculated with Streptomyces sp. PGPA39 contained lower proline content than non-inoculated tomatoes but exhibited higher dry biomass and chlorophyll content under salinity stress $(180 \mathrm{mM} \mathrm{NaCl})$. Similarity, inoculation and co-inoculation of Arthrobacter strain MS-1 and MS-2 with symbiotic bacteria (Sinorhizobium meliloti strains R1 and R2) decreased proline content in leaves and improved growth rate, number of nodules, and salt tolerance in alfalfa under salinity stress $(100 \mathrm{mM} \mathrm{NaCl})$ [39]. Therefore, our results suggested that $D$. barathri MT2. $1^{\mathrm{T}}$ may use another alternative approach in combination with proline accumulation to alleviate salinity stress.

Salinity stress increases ROS in plant cells including $\mathrm{H}_{2} \mathrm{O}_{2}$ accumulation which affects several plant physiology in particular stomatal closure $[33,94]$. Stomatal closure was suggested to play role in plant adaptation during stress by preventing water loss through transpiration [94]. Chloroplast ultrastructure destruction, chlorophyll malfunction, and chlorophyll content reduction were also reported, which ultimately lowered or inhibited photosynthesis of plants [16]. Tomato seedlings inoculated with deep-sea actinobacteria showed higher chlorophyll content than control seedlings and demonstrated a positive effect on the growth and development of tomato seedlings in terms of shoot length, root length, and dry weight for D. barathri MT2.1 ${ }^{\mathrm{T}}$; shoot and root length for D. profundi MT2.2 ${ }^{\mathrm{T}}$. Similarly, Streptomyces sp. PGPA39-inoculated tomato showed higher total chlorophyll in leaves than non-inoculated tomato under both non-stress and salt stress $(180 \mathrm{mM} \mathrm{NaCl})$ 
conditions and improved plant dry biomass [31]. An increase in chlorophyll content was observed in mungbean (Vigna radiata $\mathrm{L}$.) inoculated with Bacillus cereus $\mathrm{Pb} 25$ under $180 \mathrm{mM} \mathrm{NaCl}$ resulting in a higher fresh and dry root and shoot weight and yield compared to control plants [93]. In addition, chlorophyll content of maize inoculated with Azotobacter spp. $\mathrm{C} 5$ and $\mathrm{C} 9$ under salinity $\left(5.85 \mathrm{~g} \mathrm{NaCl} \mathrm{kg}^{-1}\right)$ was increased and resulted in a positive effect on maize growth in terms of root and shoot length, root and shoot dry weight [95]. In contrast, inoculation of terrestrial D. nishinomiyaensis $\mathrm{DSM} 20448^{\mathrm{T}}$ resulted in decreased chlorophyll content in leaves of tomato seedlings. Salinity stress is known to induce Fe-related deficiency, including chlorosis in plants [30]. Microbial siderophores have high specificity toward Fe and play a significant role in Fe availability for plants. Iron is required for enzymes responsible for chlorophyll synthesis. Interestingly, D. barathri MT2. $1^{\mathrm{T}}$ produced the highest hydroxamate siderophore, approximately two times higher than other Dermacoccus strains. This observation corresponded well with the recorded total chlorophyll content in tomato inoculated with $D$. barathri MT2.1 $1^{\mathrm{T}}$ that was two times higher than other treatments. Indeed, siderophore-producing bacteria have been shown to confer salt tolerance in several plant species, e.g., wheat $[71,96,97]$. These previous studies and the present study results suggest the role of siderophores in mitigating salinity stress in plants.

Hydrogen peroxide $\left(\mathrm{H}_{2} \mathrm{O}_{2}\right)$ is a ROS indicator of oxidative stress in plants under various stress, including salinity. The overproduction of ROS causes cell death via various pathways, such as lipid peroxidation, protein oxidation, nucleic acid damage, and programmed cell death [98]. An increase in antioxidant enzyme activities indicates high oxidative stress in plants [99]. In the present study, $\mathrm{H}_{2} \mathrm{O}_{2}$ in leaves of tomatoes inoculated with $D$. barathri $\mathrm{MT} 2.1^{\mathrm{T}}$ and $D$. profundi $\mathrm{MT} 2.2^{\mathrm{T}}$ was lower than that in non-inoculated tomatoes under salinity (Figure 3d). Deep-sea Dermacoccus might enhance $\mathrm{H}_{2} \mathrm{O}_{2}$ scavenging enzymes, such as catalase (CAT) and peroxidase (POD), which help maintain $\mathrm{H}_{2} \mathrm{O}_{2}$ homeostasis and lead to plant growth under salt stress. Previously, inoculation of B. cereus $\mathrm{Pb} 25$ in mungbean under $9 \mathrm{dS} \mathrm{m}^{-1} \mathrm{NaCl}$ increased CAT and POD enzymes resulted in a reduction of $\mathrm{H}_{2} \mathrm{O}_{2}$ level [93]. Later, soybean inoculated with Pseudomonas sp. strain AK-1 and Bacillus sp. strain SJ-5 under $100 \mathrm{mM} \mathrm{NaCl}$ showed lower catalase activity than non-inoculated plants. However, the peroxidase enzyme observed in soybean plants inoculated with SJ-5 strain was higher than in non-inoculated plants [98]. On the contrary, D. nishinomiyaensis DSM20448 ${ }^{\mathrm{T}}$-inoculated tomato showed a similar level of $\mathrm{H}_{2} \mathrm{O}_{2}$ to that found in non-inoculated tomato plants under salt stress. This observation suggests that tomatoes inoculated with D. nishinomiyaensis DSM20448 ${ }^{\mathrm{T}}$ and non-inoculated tomatoes were encountering similar stress. Our results showed that $\mathrm{H}_{2} \mathrm{O}_{2}$ production was reduced by the inoculation of tomato seedlings with the deep-sea Dermacoccus. This data supported the view that these deep-sea Dermacoccus have the potential to alleviate salt stress in tomato seedlings by promoting $\mathrm{H}_{2} \mathrm{O}_{2}$ reduction.

\subsection{Biosafety Tests for Actinobacteria \\ 3.3.1. Pathogenicity Bioassay Based on Caenorhabditis elegans}

Biosafety of bacterial strains intended to be used as PGPB is of paramount importance before releasing the plant biostimulant [100]. Caenorhabditis elegans is an alternative model to study infectious diseases in higher organisms, including humans $[65,101,102]$. C. elegans is suitable for use as a model because $36 \%$ of its 19,000 proteins in the genome match with those in humans [101]. In the bioassay, we evaluated the virulence of Dermacoccus on the number of adults, juveniles, eggs, and the death of nematodes (C. elegans). Nematodes fed with a non-pathogenic strain (E. coli OP50) showed the highest growth and reproduction and the lowest death rate at $144 \mathrm{~h}$. In contrast, when fed with a pathogenic strain (P. aeruginosa PA14), nematodes died after $96 \mathrm{~h}$, which indicated that these strains were harmful to C. elegans. All Dermacuccus strains, except D. barathri MT2.1 ${ }^{\mathrm{T}}$, showed a negative effect on the survival and fertility of C. elegans. Nematodes fed with D. barathri MT2.1 ${ }^{\mathrm{T}}$ showed the lowest severity compared to other Dermacoccus. This strain showed a death rate of only $1.8 \%$ compared to E. coli OP50 (1.6\%). D. nishinomiyaensis DSM20448 ${ }^{\mathrm{T}}(23 \%)$ and 
D. profundi $\mathrm{MT} 2.2^{\mathrm{T}}(11 \%)$ showed a higher death rate than D. barathri MT2.1 ${ }^{\mathrm{T}}$. However, the death rate of all Dermacoccus strains was lower than that of the pathogenic P. aeruginosa PA14. This observation suggested that D. barathri MT2.1 $1^{\mathrm{T}}$ is considered to be safer than other Dermacoccus strains.

\subsubsection{Escherichia coli MC4100 Sensitivity}

A sensitivity test of E. coli MC4100 viability was performed to assess harmful products release from marine actinobacteria, which were used as plant growth-promoting actinobacteria on soil microbial communities. We have shown that the survival rate of E. coli MC4100 exposed to supernatants from two marine actinobacteria, D. barathri MT2.1 $1^{\mathrm{T}}$ and $D$. profundi MT2. $2^{\mathrm{T}}$ was similar to the rate of non-pathogenic P. putida KT2440. Our results suggested that no deleterious effects on microbial communities occur when secondary metabolites produced by these marine Dermacoccus are released in soils.

\subsubsection{Ecotoxicity Test in Earthworms (Eisenia foetida)}

Earthworms are important organisms in the soil and are a key indicator of ecosystem health [103]. Assessment of the risk that PGPB may have on earthworms is necessary. Therefore, we investigated the potential effects of Dermacoccus on earthworm (E. foetida) development. Earthworms fed with chickpea supplemented with P. putida KT2440, D. nishinomiyaensis DSM20448 ${ }^{\mathrm{T}}$, and D. barathri MT2.1 $1^{\mathrm{T}}$ showed a similar growth pattern regarding total weight and length compared to control chickpea. A similar number of eggs was recorded in all treatments with bacteria, being the lowest number observed in the negative control $(2 \% \mathrm{NaCl})$. These results suggest that our Dermacoccus strains were safe for soil organisms, at least in earthworms.

\subsubsection{Daphnia magna Toxicity Bioassay}

Daphnia magna is a planktonic crustacean used as a marker for toxicity screening of bacterial substances in the aquatic ecosystem [65]. Assay reports as the EC50 value (the concentration of bacterial supernatants that can kill $50 \%$ of D. magna). The EC50 value of supernatants from Dermacoccus strains was higher than EC50 from the pathogenic strain B. cepacia CC-A174. This observation supports the safety of using Dermacoccus strains in the aquatic ecosystem. Low EC50 value indicated a high degree of toxicity on D. magna as exemplified in B. cepacia CC-A174 [65]. These results in conjunction with those observed in C. elegans would suggest that $D$. barathri MT2.1 $1^{\mathrm{T}}$ is safe enough for its use as a plant biostimulant for their protection against salinity. However, additional biosafety tests are needed to recommend the safe use of $D$. profundi $\mathrm{MT} 2 \cdot 2^{\mathrm{T}}$.

\section{Materials and Methods}

\subsection{Bacterial Strains}

Two deep-sea actinobacteria isolated from Mariana Trench sediments in the western Pacific Ocean: D. barathri MT2.1 ${ }^{\mathrm{T}}$ [64], and D. profundi MT2.2 ${ }^{\mathrm{T}}$ [64] and type species of the genus, D. nishinomiyaensis DSM20448 ${ }^{\mathrm{T}}$, were included in this study for plant experiment. Burkholderia cepacia CC-A174, Escherichia coli OP50, E. coli MC4100, Pseudomonas aeruginosa PA14, and P. putida KT2440 were used for biosafety tests. All bacterial strains were routinely cultivated on tryptic soy agar (TSA) (Difco BBL, Sparks, MD, USA) and tryptic soy broth (TSB) (Difco BBL, Sparks, MD, USA) at room temperature.

\subsection{Plant Growth-Promoting Properties In Vitro}

- Indole-3-acetic acid (IAA)

The IAA concentration was determined by the standard colorimetric method described by Lasudee et al. [68] with some modifications. Actinobacteria (1 loop full) were grown in glucose yeast extract broth (glucose $10 \mathrm{~g}$, yeast extract $10 \mathrm{~g}$, and distilled water $1000 \mathrm{~mL}$ ) supplemented with L-tryptophan $\left(2 \mathrm{mg} \mathrm{mL}^{-1}\right)$ on a shaker at $110 \mathrm{rpm}$ for 7 days in the dark. The following treatments were investigated: (1) control (No NaCl), (2) $150 \mathrm{mM} \mathrm{NaCl}$, 
(3) $300 \mathrm{mM} \mathrm{NaCl}$ and (4) $450 \mathrm{mM} \mathrm{NaCl}$. After 7 days, the supernatant was collected by centrifugation at 11,000 rpm for $5 \mathrm{~min}$. The supernatant $(1 \mathrm{~mL})$ was mixed with $2 \mathrm{~mL}$ of Salkowski's reagent [104] and incubated at room temperature for $30 \mathrm{~min}$. IAA production was detected spectrophotometrically by measuring the absorbance at $530 \mathrm{~nm}$. The quantity of IAA production was determined from a standard curve.

- $\quad$ Siderophores

Siderophore production was determined using chrome azurol S (CAS) agar [105]. Each actinobacterium (1 loop full) was cultured on TSA at room temperature for 3 days. Actinobacteria were inoculated on CAS agar and incubated at $28^{\circ} \mathrm{C}$ for 7 days in the dark. The yellow or orange halo zone around the agar plugs on a blue background on CAS agar indicates siderophore production. Quantitative analysis of siderophore production was performed by growing each actinobacterium in King's B broth (proteose peptone\#3 $10 \mathrm{~g}$, glycerol $10 \mathrm{~mL}, \mathrm{~K}_{2} \mathrm{HPO}_{4} 1.5 \mathrm{~g}, \mathrm{MgSO}_{4} 1.5 \mathrm{~g}$, and distilled water $1000 \mathrm{~mL}$ ) on a shaker at $110 \mathrm{rpm}$ for 7 days. Hydroxamate type and catecholate type siderophores were detected by ferric perchlorate assay [106] and Arnow assay [107], respectively.

- Phosphate Solubilization

Screening of phosphate solubilizing activity was determined on Pikovskaya's agar (PVK) supplemented with $0.5 \%(w / v)$ tri-calcium phosphate [108]. Each actinobacterium (1 loop full) was cultured on TSA at room temperature for 3 days. Actinobacteria were inoculated on PVK agar and incubated at $28{ }^{\circ} \mathrm{C}$ for 7 days. A clear zone of tri-calcium phosphate solubilization around the agar plugs indicates phosphate solubilization. For quantifying the released phosphorus, actinobacteria were grown in Pikovskaya's broth (glucose $10 \mathrm{~g}$, tri-calcium phosphate $5 \mathrm{~g}, \mathrm{NaCl} 0.2 \mathrm{~g}, \mathrm{KCl} 0.2 \mathrm{~g}, \mathrm{MgSO}_{4} 0.1 \mathrm{~g}, \mathrm{MnSO}_{4}$ $0.0025 \mathrm{~g}, \mathrm{Fe}_{2}\left(\mathrm{SO}_{4}\right)_{3} 0.0025 \mathrm{~g}$, $\left(\mathrm{NH}_{4}\right)_{2} \mathrm{SO}_{4} 0.5 \mathrm{~g}$, and distilled water $\left.1000 \mathrm{~mL}\right)$ supplemented with $0.5 \%(w / v)$ tri-calcium phosphate on a shaker at $110 \mathrm{rpm}$ at room temperature for 7 days. The supernatant was collected to determine phosphorus released in culture broth using a colorimetric method [109].

- 1-Aminocyclopropane-1-Carboxylate (ACC) Deaminase Activity

ACC deaminase activity was detected based on the method of Palaniyandi et al. [31]. All actinobacteria were grown on a minimal medium [110] without nitrogen source (negative control), minimal medium supplemented with ammonium sulfate (positive control), and minimal medium supplemented with ACC at a final concentration of $3 \mathrm{mmol} \mathrm{L}^{-1}$. The incubation of all agar plates was performed at $30^{\circ} \mathrm{C}$ for 7 days. Strains able to grow on ACC as nitrogen source exhibit a similar growth to those cultured in $\left(\mathrm{NH}_{4}\right)_{2} \mathrm{SO}_{4}$ containing medium (positive ACC deaminase), whereas strains that could not utilize ACC show similar growth to those cultured in a medium without a nitrogen source (negative ACC deaminase).

\subsection{Preparation of Inoculum for Plant Experiments}

All actinobacteria were grown in TSB at room temperature on a shaker at $110 \mathrm{rpm}$ for 48-72 h. Cells were collected by centrifugation at $5000 \mathrm{rpm}$ for $15 \mathrm{~min}$. Then, cells were resuspended in $0.5 \mathrm{X}$ M9 buffer and adjusted to $10^{8}-10^{9} \mathrm{CFU} / \mathrm{mL}$, as described by Narváez-Reinaldo et al. [111].

\subsection{Enhancement of Tomato Seedlings Growth without Salt Stress}

This experiment is designed to test the growth-promoting ability of Dermacoccus on tomato seedlings without salt stress. Seedlings of 1-month-old tomato (Solanum lycopersicum, Raf tomato) were purchased from SaliPlant S.L. specialist grower (Granada, Spain), were planted in the pot, containing a mixture of $50 \%(v / v)$ plant substrate and $50 \%(v / v)$ vermiculite. The following treatments were prepared: (1) control (non-inoculated) $3 \mathrm{~mL}$ M9 buffer were added, (2) D. barathri MT2.1 ${ }^{\mathrm{T}}$, (3) D. profundi MT2. $2^{\mathrm{T}}$, and (4) D. nishinomiyaensis DSM20448 ${ }^{\mathrm{T}}$ suspension. Each actinobacterial suspension $(3 \mathrm{~mL})$ contained $10^{8}-10^{9} \mathrm{CFU} / \mathrm{mL}$ 
in 0.5X M9 buffer was added per seedling. Tomato seedlings were watered regularly until the end of the experiment. The following parameters were performed at days 0 and 26: shoot length, root length, fresh weight, and dry weight [112].

\subsection{Enhancement of Tomato Seedlings Growth under Salt Stress}

All Dermacoccus strains that promoted tomato growth without salt stress were selected to test their protective effect on tomato seedlings under salt stress. Seedlings of 1-month-old tomato (Solanum lycopersicum, Raf tomato) from SaliPlant S.L. specialist grower (Granada, Spain) were planted in trays size $2.5 \times 2.5 \times 7 \mathrm{~cm}$ and were regularly watered for 7 days. The pots were incubated in a growth room at a constant relative humidity (50-60\%). The room was lit with a 12-h day/night cycle and gradual dimming/brightening of the light to simulate dawn and dusk. The day cycle consisted of $200 \mu \mathrm{mol}$ photons $\cdot \mathrm{m}^{-2} \cdot \mathrm{s}^{-1}$, and the dawn-dusk cycle consisted of $150 \mu \mathrm{mol}$ photons $\cdot \mathrm{m}^{-2} \cdot \mathrm{s}^{-1}$. The temperature was programmed to change from 18 to $20^{\circ} \mathrm{C}$ for the night cycle to $20-25^{\circ} \mathrm{C}$ in the diurnal [57]. Salt stress condition was applied to the seedlings by adding $150 \mathrm{mM} \mathrm{NaCl}$ solution, and this was considered as day 0 of the experiment. Electrical conductivity (EC1:5) was increased from $0.329 \pm 0.02 \mathrm{dS} \mathrm{m}^{-1}$ in absence of $\mathrm{NaCl}$ to $1.158 \pm 0.04 \mathrm{dS} \mathrm{m}^{-1}$ for samples containing $150 \mathrm{mM} \mathrm{NaCl}$. The following treatments were investigated: (1) control (non-inoculated with stress) $0.5 \mathrm{X}$ M9 buffer was added and (2) actinobacterial suspension $\left(10^{8}-10^{9} \mathrm{CFU} / \mathrm{mL}\right.$ in $0.5 \mathrm{X}$ M9 buffer). The actinobacterial suspensions prepared as described in 4.3 were applied at the rate of $2 \mathrm{~mL} /$ seedling by means of pouring. In addition, non-stressed treatment without bacterial inoculation was also carried out for comparison purposes. The following parameters were investigated after 14 days of inoculation: fresh weight (FW), root length, shoot length. Turgid weight (TW) was recorded after soaking the roots in distilled water for $48 \mathrm{~h}$ in the dark at room temperature. Dry weight (DW) was recorded after drying plants in the oven at $60^{\circ} \mathrm{C}$ until constant weight [112]. Relative water content (RWC) was estimated according to the formula $\{$ RWC $(\%)=[(F W-D W) /(T W-D W) \times 100]\}[113]$. Membrane stability index (MSI) was determined according to Asharf et al. [28]. Briefly, leaves of tomato $(0.1 \mathrm{~g})$ were washed in running tap water, washed in $10 \mathrm{~mL}$ distilled water, and heated at $40{ }^{\circ} \mathrm{C}$ for $30 \mathrm{~min}$. The electrical conductivity was recorded (C1). The same sample was boiled in a water bath at $100{ }^{\circ} \mathrm{C}$ for $10 \mathrm{~min}$, and electrical conductivity was recorded $(\mathrm{C} 2)$. MSI was calculated as MSI $=[1-(\mathrm{C} 1 / \mathrm{C} 2)] \times 100$.

\section{Biochemical Analysis}

Proline content in plants was assayed using a colorimetric method according to Bates et al. [113]. Tomato leaves $(250 \mathrm{mg}$ ) were crushed by pestle and mortar with $3 \mathrm{~mL}$ $95 \%$ ethanol. Supernatants were collected by centrifugation at $1500 \mathrm{rpm}$ for $5 \mathrm{~min}$. The supernatant of each sample $(200 \mu \mathrm{L})$ was mixed with $300 \mu \mathrm{L}$ distilled water and $2 \mathrm{~mL}$ of ninhydrin reagent. The mixture was boiled at $100{ }^{\circ} \mathrm{C}$ for $1 \mathrm{~h}$ and the reaction was stopped on an ice bath. After that, $6 \mathrm{~mL}$ of toluene were added and vortexed for $20 \mathrm{~s}$. The top organic layer was measured spectrophotometrically at $520 \mathrm{~nm}$. Proline content was determined from the standard curve.

Chlorophyll content was determined according to Arnon [114]. Leaf samples (100 mg; W) were ground with $2.5 \mathrm{~mL}$ acetone $(\mathrm{V})$, then centrifuged at $5000 \mathrm{rpm}$ for $10 \mathrm{~min}$. The optical density of supernatants was measured at 480,663 , and $645 \mathrm{~nm}$ by spectrophotometry. Chlorophyll content was calculated according to the following equation:

$$
\text { Total chlorophyll }\left(\mathrm{mg} \mathrm{g}^{-1}\right)=20.2 \times \text { OD645 }+8.02 \times \text { OD663 } \times \mathrm{V} /(1000 \times \mathrm{W})
$$

Total soluble sugar content (TSS) was estimated according to Dubois et al. [115]. Leaf samples $(10 \mathrm{mg}$ ) were crushed with $1 \mathrm{~mL} 80 \%$ ethanol using mortar and pestle and incubated in a water bath at $75^{\circ} \mathrm{C}$ for $15 \mathrm{~min}$. Supernatants were collected by centrifugation at $12,000 \mathrm{rpm}$ at $4{ }^{\circ} \mathrm{C}$ for $15 \mathrm{~min} ; 250 \mu \mathrm{L}$ of each supernatant sample was mixed with $250 \mu \mathrm{L}$ of $80 \%$ ethanol, $2.5 \mathrm{~mL}$ of concentrated sulfuric acid, and $0.5 \mathrm{~mL}$ of $5 \%(w / v)$ phenol. The 
mixtures were kept for $20 \mathrm{~min}$ at room temperature; then, the optical density was measured at $520 \mathrm{~nm}$ with a spectrophotometer. TSS content was determined from the standard curve.

The concentration of hydrogen peroxide $\left(\mathrm{H}_{2} \mathrm{O}_{2}\right)$ was examined according to Velikova et al. [116]. Leaf samples (100 mg) were ground with $3 \mathrm{~mL} 0.1 \%(w / v)$ of trichloroacetic acid in an ice bath and centrifuged at $10,000 \mathrm{rpm}$ at $4{ }^{\circ} \mathrm{C}$ for $15 \mathrm{~min}$. Each supernatant was mixed with $0.5 \mathrm{~mL}$ of $10 \mathrm{mM}$ potassium phosphate buffer $(\mathrm{pH} 7.0)$ and $1 \mathrm{~mL}$ of $1 \mathrm{M}$ potassium iodine. The optical density was measured at $390 \mathrm{~nm}$. The quantity of $\mathrm{H}_{2} \mathrm{O}_{2}$ was determined from the standard curve.

\subsection{Biosafety Tests for Actinobacteria \\ 4.6.1. Pathogenicity Bioassay Based on Caenorhabditis elegans}

This bioassay was performed as described by Vilchez et al. [65]. Each actinobacteria culture broth was dropped in a T-shaped line $(100 \mu \mathrm{L})$ on five potatoes dextrose agar (PDA) plates. Plates were kept at $30{ }^{\circ} \mathrm{C}$ for $3 \mathrm{~h}$ to dry. Plates were maintained at $22{ }^{\circ} \mathrm{C}$ for $24 \mathrm{~h}$ before use. Five juvenile nematodes at larval stage L3-L4 were added to each plate. Plates were incubated at $22{ }^{\circ} \mathrm{C}$, and the number of adults, juveniles, deposited eggs, and dead nematodes were counted every $24 \mathrm{~h}$ for 7 days. Pseudomonas aeruginosa PA14 was used as a positive control of pathogenic strains and Escherichia coli OP50 as a positive control to estimate the natural growth and death rate of nematodes.

\subsubsection{Escherichia coli MC4100 Sensitivity}

This bioassay was performed following the method described by Vilchez et al. [65]. Cell suspension of E. coli MC4100 in M9 buffer $\left(10^{8}-10^{9} \mathrm{CFU} / \mathrm{mL}\right)(5 \mathrm{~mL})$ was mixed with $1 \mathrm{~mL}$ filtered sterile supernatant of a tested strain of actinobacteria. The mixture was incubated at room temperature for $1 \mathrm{~h}$, then serially diluted and plated on TSA to estimate the number of surviving E. coli MC4100 (CFU/mL). E. coli suspension mixed with Pseudomonas putida KT2440 supernatant was used as a non-pathogenic strain and P. aeruginosa PA14 as a pathogenic strain [102,117].

\subsubsection{Ecotoxicity Test in Earthworms (Eisenia foetida)}

Earthworm reproduction test using E. foetida was performed according to the Organisation for Economic Co-operation and Development (OCDE) Test Guideline No. 222 [118,119] with slight modifications [65]. Actinobacteria were cultured in TSB at $30{ }^{\circ} \mathrm{C}$ for $72 \mathrm{~h}$. Actinobacterial cells were collected by centrifugation, mixed with $20 \mathrm{~mL}$ of $1 \mathrm{M}$ trehalose, and freeze-dried. Ten of same generation earthworms (at least 1 month old and about 5.5-6 cm long) were put in pots filled with $0.5 \mathrm{~L}$ of a mixture of vegetal substrate and sphagnum peat $(3: 1 w / w)$ and kept at $\mathrm{pH} 6.7-7.4,70 \%$ relative humidity and $20-25^{\circ} \mathrm{C}$ for 28 days. The earthworms were fed with $5 \mathrm{~g}$ chickpea per week as a control, $4.5 \mathrm{~g}$ chickpea mixed with $0.5 \mathrm{~g}$ B. cepacia CC-A174 as a pathogenic strain, and P. putida KT2440 as a non-pathogenic strain. For testing ecotoxicity, $0.5 \mathrm{~g}$ dry powder of each actinobacterium was mixed with $4.5 \mathrm{~g}$ chickpea and fed to earthworms. As a control for the earthworm death rate, $\mathrm{NaCl}$ $(2 \% w / w)$ was added to the substrate mixture as a dry powder [57]. Length, weight, the number of ootheca, and the number of juveniles were recorded at $7,14,21$, and 28 days and expressed as an average value of ten earthworms.

\subsubsection{Daphnia magna Toxicity Bioassay}

The toxicity of actinobacterial supernatants to D. magna was tested with DaphToxKitFR (Microbiotests BE) [120] accordingly to ISO6341 and OECD Test Guideline no. 211 [121]. The ephippia were placed in standard fresh water and incubated at $20{ }^{\circ} \mathrm{C}$ for $72 \mathrm{~h}$ under continuous illumination. Twenty newly hatched daphnia were transferred to a multiwell microplate system with cell-free supernatant (10 mL/well; 5 daphnias/well). Cell-free supernatant from each actinobacterium was diluted in standard freshwater according to the manufacturer's instructions. B. cepacia CC-A174 was used as a pathogenic strain and P. putida KT2440 as a non-pathogenic strain. Immobile cells were counted at 24 and $48 \mathrm{~h}$. 


\subsection{Data Analysis}

The in vitro and in vivo plant growth-promoting experiments were performed in triplicate and presented as mean values and standard deviations (SD). The data were analyzed using a one-way analysis of variance (ANOVA) and Duncan's multiple range tests. The statistical analysis was performed using SPSS (version 17.0) at $p<0.05$. Significantly different mean values were indicated with different letters.

\section{Conclusions}

In conclusion, our results clearly show that deep-sea, D. barathri $\mathrm{MT} 2.1^{\mathrm{T}}$ could promote the growth of tomato (Solanum lycopersicum) seedlings under salinity stress. Adverse effects of salt stress in tomato seedlings were reduced as observed in various growth parameters: shoot and root length, fresh and dry weight. These deep-sea Dermacoccus may reduce the oxidative stress in tomatoes under salt stress via hydrogen peroxide reduction, possibly by the production of $\mathrm{H}_{2} \mathrm{O}_{2}$ scavenging enzymes. Biosafety assay also suggested the safety of some deep-sea Dermacoccus strains in aquatic and terrestrial ecosystems. The different results observed in the biosafety level of the various studied strains would point to the need to include this type of assays for the various Dermacoccus species. This study provides the first supporting evidence on the potential of deep-sea actinobacteria to promote the growth of plants under salinity stress.

Supplementary Materials: The following are available online at https://www.mdpi.com/article/ 10.3390/plants10081687/s1, Figure S1: Growth of tomato seedlings inoculated with Dermacoccus under $150 \mathrm{mM} \mathrm{NaCl}$. (a) Non-inoculated tomato without salt stress; (b) Non-inoculated tomato with salt stress; (c) D. nishinomiyaensis DSM20448 ${ }^{\mathrm{T}}$-inoculated tomato seedling; (d) D. barathri MT2.1 $1^{\mathrm{T}}$ inoculated tomato seedling; (e) D. profundi MT2.2 $2^{\mathrm{T}}$-inoculated tomato seedling; Figure S2: ACC deaminase activity of Dermacoccus strains. (a) Dermacoccus grown on minimal medium supplemented with $\left(\mathrm{NH}_{4}\right)_{2} \mathrm{SO}_{4}$; (b) Dermacoccus grown on minimal medium supplemented with ACC; (c) Dermacoccus grown on minimal medium.

Author Contributions: Investigation, P.R. and A.B.-R.; writing-original draft preparation, P.R. and W.P.-a.; conceptualization, W.P.-a.; funding acquisition, supervision, and writing-review and editing W.P.-a. and M.M. All authors have read and agreed to the published version of the manuscript.

Funding: This research study was funded by the Spanish Ministry for Economy and Competitiveness and the European Union, within the context of the research project CGL2017-91737-EXP and by the Andalusian Regional Government and the European Union (research project P18-RT-976) and by the European Union through the Erasmus+ program and partially supported by Chiang Mai University. PR is grateful to the Graduate School, Chiang Mai University, for the TA/RA scholarship for 2019-2021.

Institutional Review Board Statement: Not applicable.

Informed Consent Statement: Not applicable.

Data Availability Statement: Data sharing does not apply to this article as no datasets were generated or analyzed during the current study.

Conflicts of Interest: The authors declare no conflict of interest.

\section{References}

1. Dhakal, D.; Pokhrel, A.R.; Shrestha, B.; Sohng, J.K. Marine rare actinobacteria: Isolation, characterization, and strategies for harnessing bioactive compounds. Front. Microbiol. 2017, 8, 1106. [CrossRef]

2. Kamjam, M.; Sivalingam, P.; Deng, Z.; Hong, K. Deep sea actinomycetes and their secondary metabolites. Front. Microbiol. 2017, 8, 760. [CrossRef] [PubMed]

3. Pathom-aree, W.; Stach, J.E.M.; Ward, A.C.; Horikoshi, K.; Bull, A.T.; Goodfellow, M. Diversity of actinomycetes isolated from Challenger deep sediment (10,898 m) from the Mariana Trench. Extremophiles 2006, 10, 181-189. [CrossRef] [PubMed]

4. Subramani, R.; Aalbersberg, W. Marine actinomycetes: An ongoing source of novel bioactive metabolites. Microbiol. Res. 2012, 167, 571-580. [CrossRef] 
5. Das, S.; Lyla, P.S.; Khan, S.A. Marine microbial diversity and ecology: Importance and future perspectives. Curr. Sci. 2006, 90, 1325-1335.

6. Shukla, P.S.; Agarwal, P.K.; Jha, B. Improved salinity tolerance of Arachis hypogaea (L.) by the interaction of halotolerant plantgrowth-promoting rhizobacteria. J. Plant Growth Regul. 2012, 31, 195-206. [CrossRef]

7. Pathom-aree, W.; Nogi, Y.; Sutcliffe, I.C.; Ward, A.C.; Horikoshi, K.; Bull, A.T.; Goodfellow, M. Dermacoccus abyssi sp. nov., a piezotolerant actinomycetes isolated from the Mariana Trench. Int. J. Syst. Evol. Microbiol. 2006, 56, 1233-1237. [CrossRef]

8. Shahid, S.A.; Zaman, M.; Heng, L. Soil salinity: Historical perspectives and a world overview of the problem. In Guideline for Salinity Assessment, Mitigation and Adaptation Using Nuclear and Related Techniques; Zaman, M., Shahid, S.A., Heng, L., Eds.; Springer International Publishing: Cham, Switzerland, 2018; pp. 43-53.

9. Flowers, T.J.; Galal, H.K.; Bromham, L. Evolution of halophytes: Multiple origins of salt tolerance in land plants. Funct. Plant Biol. 2010, 37, 604-612. [CrossRef]

10. Qadir, M.; Tubeileh, A.; Akhtar, J.; Larbi, A.; Minhas, P.S.; Khan, M.A. Productivity enhancement of salt-affected environments through crop diversification. Land Degrad. Dev. 2008, 19, 429-453. [CrossRef]

11. Hurie, T.; Karahara, I.; Katsuhara, M. Salinity tolerance mechanisms in glycophytes: An overview with the central focus on rice plants. Rice 2012, 5, 11. Available online: http:/ / www.thericejournal.com/content/5/1/11 (accessed on 21 January 2021). [CrossRef]

12. Orozco-Mosqueda, M.D.C.; Glick, B.R.; Santoyo, G. ACC deaminase in plant growth-promoting bacteria (PGPB): An efficient mechanism to counter salt stress in crops. Microbiol. Res. 2020, 235, 126439. [CrossRef]

13. Ansari, M.; Shekari, F.; Mohammadi, M.H.; Juhos, K.; Végvári, G.; Biró, B. Salt-tolerant plant growth-promoting bacteria enhanced salinity tolerance of salt-tolerant alfalfa (Medicago sativa L.) cultivars at high salinity. Acta Physiol. Plant. 2019, 41, 195. [CrossRef]

14. Shrivastava, P.; Kumar, R. Soil salinity: A serious environmental issue and plant growth promoting bacteria as one of the tools for its alleviation. Saudi J. Biol. Sci. 2015, 22, 123-131. [CrossRef]

15. Yang, J.; Kloepper, J.W.; Ryu, C.M. Rhizosphere bacteria help plants tolerate abiotic stress. Trends Plant Sci. 2009, 14, 1-4. [CrossRef]

16. Kamran, M.; Parveen, A.; Ahmar, S.; Malik, Z.; Hussain, S.; Chattha, M.S.; Saleem, M.H.; Adil, M.; Heidari, P.; Chen, J.T. An overview of hazardous impacts of soil salinity in crops, tolerance mechanisms, and amelioration through selenium supplementation. Int. J. Mol. Sci. 2020, 21, 148. [CrossRef]

17. Kumar, A.; Verma, J.P. Does plant-microbe interaction confer stress tolerance in plants: A review. Microbiol. Res. 2018, $207,41-52$. [CrossRef]

18. Mahajan, S.; Tuteja, N. Cold, salinity and drought stresses: An overview. Arch. Biochem. Biophys. 2005, 444, 139-158. [CrossRef]

19. Yildirim, E.; Taylor, A.G.; Spittler, T.D. Ameliorative effects of biological treatments on growth of squash plants under salt stress. Sci. Hortic. 2006, 111, 1-6. [CrossRef]

20. Anwar-ul-Haq, M.; Akram, S.; Akhtar, J.; Saqib, M.; Saqib, Z.A.; Abbasi, G.H.; Jan, M. Morpho-physiological characterization of sunflower genotypes (Helianthus annuus L.) under saline condition. Pak. J. Agric. Sci. 2013, 50, 49-54.

21. Kapoor, K.; Srivastava, A. Assessment of salinity tolerance of Vigna mungo var. Pu-19 using ex vitro and in vitro methods. Asian J. Biotechnol. 2010, 2, 73-85. [CrossRef]

22. Mallahi, T.; Saharkhiz, M.J.; Javanmardi, J. Salicylic acid changes morpho-physiological attributes of feverfew (Tanacetum parthenium L.) under salinity stress. Acta Ecol. Sin. 2018, 38, 351-355. [CrossRef]

23. Petretto, G.L.; Urgeghe, P.P.; Massa, D.; Melito, S. Effect of salinity (NaCl) on plant growth, nutrient content, and glucosinolate hydrolysis products trends in rocket genotypes. Plant Physiol. Biochem. 2019, 141, 30-39. [CrossRef]

24. Ahmadi, F.; Karimi, K.; Struik, P. Effect of exogenous application of methyl jasmonate on physiological and biochemical characteristics of Brassica napus L. cv. Talaye under salinity stress. S. Afr. J. Bot. 2017, 115, 5-11. [CrossRef]

25. Jha, Y.; Subramanian, R.B. PGPR regulate caspase-like activity, programmed cell death, and antioxidant enzyme activity in paddy under salinity. Physiol. Mol. Biol. Plants 2014, 20, 201-207. [CrossRef]

26. Miransari, M.; Smith, D.L. Plant hormones and seed germination. Environ. Exp. Bot. 2014, 99, 110-121. [CrossRef]

27. Santoyo, G.; Strathern, J.N. Non-homologous end joining is important for repair of Cr (VI)-induced DNA damage in Saccharomyces cerevisiae. Microbiol. Res. 2008, 163, 113-119. [CrossRef]

28. Ashraf, M.; Shahzad, M.; Akhtar, N.; Imtiaz, M.; Ali, A. Salinization/sodification of soil and physiological dynamics of sunflower irrigated with saline-sodic water amending by potassium and farm yard manure. J. Water Reuse Desalin. 2017, 7, 476-487. [CrossRef]

29. Munns, R.; Tester, M. Mechanisms of salinity tolerance. Annu. Rev. Plant Biol. 2008, 59, 651-681. [CrossRef] [PubMed]

30. Kumar, A.; Singh, S.; Gaurav, A.K.; Srivastava, S.; Verma, J.P. Plant growth-promoting bacteria: Biological tools for the mitigation of salinity stress in plants. Front. Microbiol. 2020, 11, 1216. [CrossRef]

31. Palaniyandi, S.A.; Damodharan, K.; Yang, S.H.; Suh, J.W. Streptomyces sp. strain PGPA39 alleviates salt stress and promotes growth of 'Micro Tom' tomato plants. J. Appl. Microbiol. 2014, 117, 766-773. [CrossRef] [PubMed]

32. Taffouo, V.D.; Wamba, O.F.; Youmbi, E.; Nono, G.V.; Akoa, A. Growth, yield, water status and ionic distribution response of three bambara groundnut (Vigna subterranean (L.) verdc.) landraces grown under saline conditions. Int. J. Bot. 2010, 6, 53-58. [CrossRef]

33. Machado, R.M.A.; Serralheiro, R.P. Soil salinity: Effect on vegetable crop growth. Management practices to prevent and mitigate soil salinization. Hortoculturae 2017, 3, 30. [CrossRef] 
34. Zaman, M.; Shabbir, A.; Shahid, S.A.; Heng, L. Irrigation systems and zones of salinity development. In Guideline for Salinity Assessment, Mitigation and Adaptation Using Nuclear and Related Techniques; Zaman, M., Shahid, S.A., Heng, L., Eds.; Springer International Publishing: Cham, Switzerland, 2018; pp. 91-112.

35. Hanin, M.; Ebel, C.; Ngom, M.; Laplaze, L.; Masmoudi, K. New insights on plant salt tolerance mechanisms and their potential use for breeding. Front. Plant Sci. 2016, 7, 1787. [CrossRef]

36. Del Amor, F.M.; Cuadra-Crespo, P. Plant growth-promoting bacteria as a tool to improve salinity tolerance in sweet pepper. Funct. Plant Biol. 2012, 39, 82-90. [CrossRef]

37. Gontia-Mishra, I.; Sapre, S.; Sharma, A.; Tiwari, S. Amelioration of drought tolerance in wheat by the interaction of plant growth-promoting rhizobacteria. Plant Biol. 2016, 18, 992-1000. [CrossRef]

38. Manzanera, M. Dealing with water stress and microbial preservation. Environ. Microbiol. 2021, 23, 3351-3359. [CrossRef] [PubMed]

39. Saidi, S.; Cherif-Silini, H.; Bouket, A.C.; Silini, A.; Eshelli, M.; Luptakova, L.; Alenezi, F.N.; Belbahri, L. Improvement of Medicago sativa crops productivity by the co-inoculation of Sinorhizobium meliloti-actinobacteria under salt stress. Curr. Microbiol. 2021, 78, 1344-1357. [CrossRef] [PubMed]

40. Wang, Y.; Zhang, Z.; Zhang, P.; Cao, Y.; Hu, T.; Yang, P. Rhizobium symbiosis contribution to short-term salt stress tolerance in alfalfa (Medicago sativa L.). Plant Soil 2016, 402, 247-261. [CrossRef]

41. Sathya, A.; Vijayabharathi, R.; Gopalakrishnan, S. Plant growth-promoting actinobacteria: A new strategy for enhancing sustainable production and protection of grain legumes. 3 Biotech 2017, 7, 102. [CrossRef]

42. Chukwuneme, C.F.; Babalola, O.O.; Kutu, F.R.; Ojuederie, O.B. Characterization of actinomycetes isolates for plant growth promoting traits and their effects on drought tolerance in maize. J. Plant Interact. 2020, 15, 93-105. [CrossRef]

43. Hamedi, J.; Mohammadipanah, F. Biotechnological application and taxonomical distribution of plant growth promoting actinobacteria. J. Ind. Microbiol. Biotechnol. 2015, 42, 157-171. [CrossRef]

44. Vilchez, J.I.; Niehaus, K.; Dowling, D.N.; Gonzalez-Lopez, J.; Manzanera, M. Protection of pepper plants from drought by Microbacterium sp. 3J1 by modulation of the plant's glutamine and $\alpha$-ketoglutarate content: A comparative metabolomics approach. Front. Microbiol. 2018, 9, 284. [CrossRef]

45. Chaiharn, M.; Theantana, T.; Pathom-aree, W. Evaluation of biocontrol activities of Streptomyces spp. against rice blast disease fungi. Pathogens 2020, 9, 126. [CrossRef]

46. Meschke, H.; Schrempf, H. Streptomyces lividans inhibits the proliferation of the fungus Verticillium dahliae on seeds and roots of Arabidopsis thaliana. Microb. Biotechnol. 2010, 3, 428-443. [CrossRef]

47. Salwan, R.; Sharma, V.; Sharma, A.; Singh, A. Molecular imprints of plant beneficial Streptomyces sp. AC30 and AC40 reveal differential capabilities and strategies to counter environmental stresses. Microbiol. Res. 2020, 235, 126449. [CrossRef]

48. Barka, E.A.; Vatsa, P.; Sanchez, L.; Gaveau-Vaillant, N.; Jacquard, C.; Klenk, H.P.; Clément, C.; Ouhdouch, Y.; van Wezel, G.P. Taxonomy, physiology, and natural products of actinobacteria. Microbiol. Mol. Biol. Rev. 2015, 80, 1-43. [CrossRef]

49. Del Pulgar, E.M.G.; Saadeddin, A. The cellulolytic system of Thermobifida fusca. Crit. Rev. Microbiol. 2014, 40, 236-247. [CrossRef]

50. Kurtböke, D.I. Biodiscovery from rare actinomycetes: An eco-taxonomical perspective. Appl. Microbiol. Biotechnol. 2012, 93, 1843-1852. [CrossRef]

51. Salwan, R.; Sharma, V. The role of actinobacteria in the production of industrial enzymes. In Future Developments in Microbial Biotechnology and Bioengineering; Singh, B.P., Gupta, V.K., Passari, A.K., Eds.; Elsevier: Amsterdam, The Netherlands, 2018; pp. 165-177. [CrossRef]

52. Gong, Y.; Bai, J.-L.; Yang, H.-T.; Zhang, W.-D.; Xiong, Y.-W.; Ding, P.; Qin, S. Phylogenetic diversity and investigation of plant growth-promoting traits of actinobacteria in coastal salt marsh plant rhizospheres from Jiangsu, China. Syst. Appl. Microbiol. 2018, 41, 516-527. [CrossRef] [PubMed]

53. Pathom-aree, W.; Kreawsa, S.; Kamjam, M.; Tokuyama, S.; Yoosathaporn, S.; Lumyong, S. Potential of selected mangrove Streptomyces as plant growth promoter and rice Bakanae disease control agent. Chiang Mai J. Sci. 2019, 46, $261-276$.

54. Dias, M.P.; Bastos, M.S.; Xavier, V.B.; Cassel, E.; Astarita, L.V.; Santarém, E.R. Plant growth and resistance promoted by Streptomyces spp. in tomato. Plant Physiol. Biochem. 2017, 118, 479-493. [CrossRef]

55. Dastager, S.G.; Deepa, C.K.; Pandey, A. Isolation and characterization of novel plant growth promoting Micrococcus sp. NII-0909 and its interaction with cowpea. Plant Physiol. Biochem. 2010, 48, 987-992. [CrossRef]

56. Cardinale, M.; Ratering, S.; Suarez, C.; Montoya, A.M.Z.; Geissler-Plaum, R.; Schnell, S. Paradox of plant growth promotion potential of rhizobacteria and their actual promotion effect on growth of barley (Hordeum vulgare L.) under salt stress. Microbiol. Res. 2015, 181, 22-32. [CrossRef]

57. Vilchez, J.I.; Garcia-Fontana, C.; Román-Naranjo, D.; González-Lopéz, J.; Manzanera, M. Plant drought tolerance enhancement by trehalose production of desiccation-tolerant microorganisms. Front. Microbiol. 2016, 7, 1577. [CrossRef]

58. Azman, A.-S.; Othman, L.; Velu, S.S.; Chan, K.-G.; Lee, L.-H. Mangrove rare actinobacteria: Taxonomy, natural compound, and discovery of bioactivity. Front. Microbiol. 2015, 6, 856. [CrossRef]

59. Hong, K.; Gao, A.-H.; Xie, Q.-Y.; Gao, H.; Zhuang, L.; Lin, H.-P.; Yu, H.-P.; Li, J.; Yao, X.-S.; Goodfellow, M.; et al. Actinomycetes for marine drug discovery isolated from mangrove soils and plants in China. Mar. Drugs 2009, 7, 24-44. [CrossRef]

60. Suksaard, P.; Pathom-aree, W.; Duangmal, K. Diversity and plant growth promoting activities of actinomycetes from mangroves. Chiang Mai J. Sci. 2017, 44, 1210-1223. 
61. Valliappan, K.; Sun, W.; Li, Z. Marine actinobacteria associated with marine organisms and their potentials in producing pharmaceutical natural products. Appl. Microbiol. Biotechnol. 2014, 98, 7365-7377. [CrossRef] [PubMed]

62. Nafis, A.; Raklami, A.; Bechtaoui, N.; El Khalloufi, F.; El Alaoui, A.; Glick, B.R.; Hafidi, M.; Kouisni, L.; Ouhdouch, Y.; Hassani, L. Actinobacteria from extreme niches in Morocco and their plant growth-promoting potentials. Diversity 2019, 11, 139. [CrossRef]

63. Ocampo-Alvarez, H.; Meza-Canales, I.D.; Mateos-Salmón, C.; Rios-Jara, E.; Rodríguez-Zaragoza, F.A.; Robles-Murguía, C.; Muñoz-Urias, A.; Hernández-Herrera, R.M.; Choix-Ley, F.J.; Becerril-Espinosa, A. Diving into reef ecosystems for land-agriculture solutions: Coral microbiota can alleviate salt stress during germination and photosynthesis in terrestrial plants. Front. Plant Sci. 2020, 11, 648. [CrossRef]

64. Pathom-aree, W.; Nogi, Y.; Ward, A.C.; Horikoshi, K.; Bull, A.T.; Goodfellow, M. Dermacoccus barathri sp. nov. and Dermacoccus profundi sp. nov., novel actinomycetes isolated from deep-sea mud of the Mariana Trench. Int. J. Syst. Evol. Microbiol. 2006, 56, 2303-2307. [CrossRef]

65. Vilchez, J.I.; Navas, A.; Gonzalez-Lopez, J.; Arcos, S.C.; Manzanera, M. Biosafety test for plant growth-promoting bacteria: Proposed environmental and human safety index (EHSI) protocol. Front. Microbiol. 2016, 6, 1514. [CrossRef]

66. Bhatti, A.A.; Haq, S.; Bhat, R.A. Actinomycetes benefaction role in soil and plant health. Microb. Pathog. 2017, 111, 458-467. [CrossRef] [PubMed]

67. Battini, F.; Cristani, C.; Giovannetti, M.; Agnolucci, M. Multifunctionality and diversity of culturable bacterial communities strictly associated with spores of the plant beneficial symbiont Rhizophagus intraradices. Microbiol. Res. 2016, 183, 68-79. [CrossRef]

68. Lasudee, K.; Tokuyama, S.; Lumyong, S.; Pathom-aree, W. Actinobacteria associated with arbuscular mycorrhizal Funneliformis mosseae spores, taxonomic characterization and their beneficial traits to plants: Evidence obtained from mung bean (Vigna radiata) and Thai jasmine rice (Oryza sativa). Front. Microbiol. 2018, 9, 1247. [CrossRef]

69. Manulis, S.; Shafrir, H.; Epstein, E.; Lichter, A.; Barash, I. Biosynthesis of indole-3-acetic acid via the indole-3-acetamide pathway in Streptomyces spp. Microbiology 1994, 140, 1045-1050. [CrossRef] [PubMed]

70. Rashad, F.M.; Fathy, H.M.; El-Zayat, A.S.; Elghonaimy, A.M. Isolation and characterization of multifunctional Streptomyces species with antimicrobial, nematicidal and phytohormone activities from marine environments in Egypt. Microbiol. Res. 2015, 175, 34-47. [CrossRef] [PubMed]

71. Sadeghi, A.; Karimi, E.; Dahaji, P.A.; Javid, M.G.; Dalvand, Y.; Askari, H. Plant growth promoting activity of an auxin and siderophore producing isolate of Streptomyces under saline soil conditions. World J. Microbiol. Biotechnol. 2012, 28, 1503-1509. [CrossRef]

72. Ellermann, M.; Arthur, J.C. Siderophore-mediated iron acquisition and modulation of host-bacterial interactions. Free Radic. Biol. Med. 2017, 105, 68-78. [CrossRef]

73. Khan, A.; Singh, P.; Srivastava, A. Synthesis, nature and utility of universal iron chelator -siderophore: A review. Microbiol. Res. 2017, 212-213, 103-111. [CrossRef]

74. Glick, B.R. Plant growth-promoting bacteria: Mechanisms and applications. Scientifica 2012, 2012, 963401. [CrossRef]

75. Marra, L.M.; de Oliveira, S.M.; Soares, C.R.F.S.; Moreira, F.M.D.S. Solubilisation of inorganic phosphates by inoculant strains from tropical legumes. Sci. Agric. 2011, 68, 603-609. [CrossRef]

76. Pérez, E.; Sulbaran, M.; Ball, M.M.; Yarzabal, L.A. Isolation and characterization of mineral phosphate solubilizing bacteria naturally colonizing a limonitic crust in the south-eastern Venezuela region. Soil Biol. Biochem. 2007, 39, 2905-2914. [CrossRef]

77. Dastager, S.; Damare, S. Marine actinobacteria showing phosphate-solubilizing efficiency in Chorao island, Goa, India. Curr. Microbiol. 2013, 66, 421-427. [CrossRef] [PubMed]

78. Zahir, Z.A.; Ghani, U.; Naveed, M.; Nadeem, S.M.; Asghar, H.N. Comparative effectiveness of Pseudomonas and Serratia sp. containing ACC-deaminase for improving growth and yield of wheat (Triticum aestivum L.) under salt-stressed conditions. Arch. Microbiol. 2009, 191, 415-424. [CrossRef] [PubMed]

79. Xiong, Y.-W.; Gong, Y.; Li, X.-W.; Chen, P.; Ju, X.-Y.; Zhang, C.-M.; Yuan, B.; Lv, Z.-P.; Xing, K.; Qin, S. Enhancement of growth and salt tolerance of tomato seedlings by a natural halotolerant actinobacterium Glutamicibacter halophytocola KLBMP 5180 isolated from a coastal halophyte. Plant Soil 2019, 445, 307-322. [CrossRef]

80. Abdel-Mageed, W.M.; Milne, B.F.; Wagner, M.; Schumacher, M.; Sandor, P.; Pathom-aree, W.; Goodfellow, M.; Bull, A.T.; Horikoshi, K.; Ebel, R.; et al. Dermacozines, a new phenazine family from deep-sea dermacocci isolated from a Mariana Trench sediment. Org. Biomol. Chem. 2010, 8, 2352-2362. [CrossRef]

81. Abdel-Mageed, W.M.; Juhasz, B.; Lehri, B.; Alqahtani, A.S.; Nouioui, I.; Pech-Puch, D.; Tabudravu, J.N.; Goodfellow, M.; Rodríguez, J.; Jaspars, M.; et al. Whole genome sequence of Dermacoccus abyssi MT1.1 isolated from the Challenger Deep of the Mariana Trench reveals phenazine biosynthesis locus and environmental adaptation factors. Mar. Drugs 2020, 18, 131. [CrossRef]

82. Karni, L.; Aktas, H.; Deveturero, G.; Aloni, B. Involvement of root ethylene and oxidative stress-related activities in preconditioning of tomato transplants by increased salinity. J. Hortic. Sci. Biotechnol. 2010, 85, 23-29. [CrossRef]

83. Bernstein, N. Effects of salinity on root growth plant roots. In Plant Roots: The Hidden Half, 4th ed.; Eshel, A., Beeckman, T., Eds.; CRC Press: Boca Raton, FL, USA, 2013; pp. 1-18.

84. Tank, N.; Saraf, M. Salinity-resistant plant growth promoting rhizobacteria ameliorates sodium chloride stress on tomato plants. J. Plant Interact. 2010, 5, 51-58. [CrossRef]

85. Hossain, Z.; Mandal, A.K.A.; Datta, S.K.; Biswas, A.K. Decline in ascorbate peroxidase activity-A prerequisite factor for tepal senescence in gladiolus. J. Plant Physiol. 2006, 163, 186-194. [CrossRef] 
86. Anjum, S.A.; Xiie, X.-Y.; Wang, L.-C.; Saleem, M.F.; Man, C.; Lei, W. Morphological, physiological and biochemical responses of plants to drought stress. Afr. J. Agric. Res. 2011, 6, 2026-2032. [CrossRef]

87. Rosa, M.; Prado, C.; Podazza, G.; Interdonato, R.; Gonzalez, J.A.; Hilal, M.; Prado, F.E. Soluble sugars-Metabolism, sensing and abiotic stress. Plant Signal Behav. 2009, 4, 388-393. [CrossRef] [PubMed]

88. Sami, F.; Yusuf, M.; Faizan, M.; Faraz, A.; Hayat, S. Role of sugars under abiotic stress. Plant Physiol. Biochem. 2016, 109, 54-61. [CrossRef] [PubMed]

89. Hu, M.; Shi, Z.; Zhang, Z.; Zhang, Y.; Li, H. Effects of exogenous glucose on seed germination and antioxidant capacity in wheat seedlings under salt stress. Plant Growth Regul. 2012, 68, 177-188. [CrossRef]

90. Wang, L.; Li, G.; Wei, S.; Li, L.J.; Zuo, S.; Liu, X.; Gu, W.; Li, J. Effects of exogenous glucose and sucrose on photosynthesis in triticale seedlings under salt stress. Photosynthetica 2019, 57, 286-294. [CrossRef]

91. Claussen, W. Proline as a measure of stress in tomato plants. Plant Sci. 2005, 168, 241-248. [CrossRef]

92. Julca, I.; Alaminos, M.; Gonzalez-Lopez, J.; Manzanera, M. Xeroprotectants for the stabilization of biomaterials. Biotechnol. Adv. 2012, 30, 1641-1654. [CrossRef]

93. Islam, F.; Yasmeen, T.; Arif, M.S.; Ali, S.; Ali, B.; Hameed, S.; Zhou, W. Plant growth promoting bacteria confer salt tolerance in Vigna radiata by up-regulating antioxidant defense and biological soil fertility. Plant Growth Regul. 2016, 80, 23-36. [CrossRef]

94. Proietti, S.; Falconieri, G.S.; Bertini, L.; Baccelli, I.; Paccosi, E.; Belardo, A.; Timperio, A.M.; Caruso, C. GLYI4 plays a role in methylglyoxal detoxification and jasmonate-mediated stress responses in Arabidopsis thatiana. Biomolecules 2019, 9, 635. [CrossRef]

95. Rojas-Tapias, D.; Moreno-Galván, A.; Pardo-Días, S.; Obando, M.; Rivera, D.; Bonilla, R. Effect of inoculation with plant growth-promoting bacteria (PGPB) on amelioration of saline stress in maize (Zea mays). Appl. Soil Ecol. 2012, 61, 264-272. [CrossRef]

96. Kavamura, V.N.; Santos, S.N.; da Silva, J.L.; Parma, M.M.; Ávila, L.A.; Visconti, A.; Zucchi, T.D.; Taketani, R.G.; Andreote, F.D.; de Melo, I.S. Screening of Brazilian cacti rhizobacteria for plant growth promotion under drought. Microbiol. Res. 2013, 168, 183-191. [CrossRef]

97. Ramadoss, D.; Lakkineni, V.K.; Bose, P.; Ali, S.; Annapurna, K. Mitigation of salt stress in wheat seedlings by halotolerant bacteria isolated from saline habitats. SpringerPlus 2013, 2, 6. [CrossRef]

98. Kumari, S.; Vaishnav, A.; Jain, S.; Varma, A.; Choudhary, D.K. Bacterial-mediated induction of systemic tolerance to salinity with expression of stress alleviating enzymes in soybean (Glycine max L. Merrill). J. Plant Growth Regul. 2015, 34, 558-573. [CrossRef]

99. Sharma, P.; Jha, A.B.; Dubey, R.S.; Pessarakli, M. Reactive oxygen species, oxidative damage, and antioxidative defense mechanism in plants under stressful conditions. J. Bot. 2012, 2012, 217037. [CrossRef]

100. Barros-Rodríguez, A.; Rangseekaew, P.; Lasudee, K.; Pathom-aree, W.; Manzanera, M. Regulatory risks associated with bacteria as biostimulants and biofertilizers in the frame of the European Regulation (EU) 2019/1009. Sci. Total Environ. 2020, 740, 140239. [CrossRef]

101. Darby, C.; Cosma, C.L.; Thomas, J.H.; Manoil, C. Lethal paralysis of Caenorhabditis elegans by Pseudomonas aeruginosa. Proc. Natl. Acad. Sci. USA 1999, 96, 15202-15207. [CrossRef]

102. García-Fontana, C.; Vilchez, J.I.; Gonzalez-Requena, M.; Gonzalez-Lopez, J.; Krell, T.; Matilla, M.A.; Manzanera, M. The involvement of McpB chemoreceptor from Pseudomonas aeruginosa PAO1 in virulence. Sci. Rep. 2019, 9, 13166. [CrossRef]

103. Nahmani, J.; Hodson, M.E.; Black, S. A review of studies performed to assess metal uptake by earthworms. Environ. Pollut. 2007, 145, 402-424. [CrossRef]

104. Glickmann, E.; Dessaux, Y. A critical examination of the specificity of the Salkowski reagent for indolic compounds produced by phytopathogenic bacteria. Appl. Environ. Microbiol. 1995, 61, 793-796. [CrossRef]

105. Schwyn, B.; Neilands, J.B. Universal chemical assay for the detection and determination of siderophores. Anal. Biochem. 1987, 160, 47-56. [CrossRef]

106. Atkin, C.L.; Neilands, J.B.; Phaff, H.J. Rhodotorulic acid, from species of Leucosporidium, Rhodosporidium, Rhodotorula, Sporodiobolus and Sporobolomyces and a new alanine containing ferrichrome from Cryptcoccus melibiosum. J. Bacteriol. 1970, 103, $722-733$. [CrossRef]

107. Arnow, L.E. Colorimetric determination of the components of 3,4-Dihidroxyphemylalanine tyrosine mixtures. J. Biol. Chem. 1937, 118, 531-537. [CrossRef]

108. Nautiya, C.S. An efficient microbiological growth medium for screening phosphate solubilizing microorganisms. FEMS Microbiol. Lett. 1999, 170, 265-270. [CrossRef]

109. Fiske, C.H.; Subbarow, Y. The colorimetric determination of phosphorus. J. Biol. Chem. 1925, 66, 375-400. [CrossRef]

110. Dworkin, M.; Foster, J.W. Experiments with some microorganisms which utilize ethane and hydrogen. J. Bacteriol. 1958, 175, 592-601. [CrossRef] [PubMed]

111. Narvaez-Reinaldo, J.J.; Barba, I.; Gonzalez-Lopez, J.; Tunnacliffe, A.; Manzanera, M. Rapid method for isolation of desiccationtolerant strains and xeroprotectants. Appl. Environ. Microbiol. 2010, 76, 5254-5262. [CrossRef] [PubMed]

112. Oukarroum, A.; El Madidi, S.; Schansker, G.; Strasser, R.J. Probing the responses of barley cultivars (Hordeum vulgare L.) by chlorophyll a fluorescence OLKJIP under drought stress and re-watering. Environ. Exp. Bot. 2007, 60, 438-446. [CrossRef]

113. Bates, L.; Waldren, B.P.; Teare, I.D. Rapid determination of free proline for water-stress studies. Plant Soil 1973, $39,205-207$. [CrossRef]

114. Arnon, D.I. Copper enzymes in isolated chloroplasts. Polyphenol oxidase in Beta vulgaris. Plant Physiol. 1949, 24, 1-15. [CrossRef] 
115. DuBois, M.; Gilles, K.A.; Hamilton, J.K.; Rebers, P.A.; Smith, F. Colorimetric method for determination of sugars and related substances. Anal. Chem. 1956, 28, 350-356. [CrossRef]

116. Velikova, V.; Yordanov, I.; Edreva, A. Oxidative stress and some antioxidant systems in acid rain-treated bean plants protective role of exogenous polyamines. Plant Sci. 2000, 151, 59-66. [CrossRef]

117. Vilchez, S.; Tunnacliffe, A.; Manzanera, M. Tolerance of plastic-encapsulated Pseudomonas putida KT2440 to chemical stress. Extremophiles 2008, 12, 297-299. [CrossRef] [PubMed]

118. Neuhauser, E.F.; Callahan, C.A. Growth and reproduction of the earthworm Eisenia foetida exposed to sublethal concentrations of organic chemicals. Soil Biol. Biochem. 1990, 22, 175-179. [CrossRef]

119. OECD. Test No. 222: Earthworm Reproduction Test (Eisenia foetida/Eisenia andrei); OECD Publishing: Paris, France, 2004.

120. Hernando, M.D.; Ejerhoon, M.; Fernandez-Alba, A.R.; Chisti, Y. Combined toxicity effects of MTBE and pesticides measured with Vibrio fischeri and Daphnia magna bioassays. Water Res. 2003, 37, 4091-4098. [CrossRef]

121. OECD. Test No. 211: Daphnia magna Reproduction Test; OECD Publishing: Paris, France, 2008. 